\title{
SUFFICIENT CONDITIONS UNDER WHICH A TRANSITIVE SYSTEM IS CHAOTIC
}

\author{
E. AKIN, E. GLASNER, W. HUANG, S. SHAO AND X. YE
}

\begin{abstract}
Let $(X, T)$ be a topologically transitive dynamical system. We show that if there is a subsystem $(Y, T)$ of $(X, T)$ such that $(X \times Y, T \times T)$ is transitive, then $(X, T)$ is strongly chaotic in the sense of $\mathrm{Li}$ and Yorke. We then show that many of the known sufficient conditions in the literature, as well as a few new results, are corollaries of this fact. In fact the kind of chaotic behavior we deduce in these results is a much stronger variant of Li-Yorke chaos which we call uniform chaos. For minimal systems we show, among other results, that uniform chaos is preserved by extensions and that a minimal system which is not uniformly chaotic is PI.
\end{abstract}

\section{Contents}

Introduction

1. Preliminary definitions and results

1.1. Transitivity and related notions

1.2. The enveloping semigroup

1.3. Some notions of Chaos

1.4. Families and filters

2. Transitivity, rigidity and proximality

2.1. Rigid and proximal sets

2.2. Transitivity implies partial rigidity

3. A criterion for chaos and applications

3.1. A criterion for chaos

3.2. Some applications

4. The Kuratowski-Mycielski Theory

4.1. The Kuratowski-Mycielski Theorem

4.2. Uniform chaos in light of the Kuratowski-Mycielski Theorem 16

5. Chaotic subsets of minimal systems 18

5.1. On the structure of minimal systems 18

5.2. Lifting chaotic sets 19

5.3. Weakly mixing extensions

5.4. The non $P I$ case 22

5.5. The proximal but not almost one-to-one case 23

5.6. The $P I$, non- $H P I$ case 25

2000 Mathematics Subject Classification. Primary: 37B05, 37B20, 54H20.

Key words and phrases. Uniform chaos, Li-Yorke chaos, Davaney chaos, Mycielski sets, enveloping semigroups, minimal systems, weak mixing, proximal, PI, point distal. 
6. Table

7. Appendix

7.1. A characterization of RIC extensions

\section{INTRODUCTION}

The presence or the lack of chaotic behavior is one of the most prominent traits of a dynamical system. However, by now there exists in the literature on dynamical systems a plethora of ways to define Chaos. In 1975, Li and Yorke introduced a notion of chaos [LY75], known now as Li-Yorke chaos, for interval maps. With a small modification this notion can be extended to any metric space. Another notion was introduced later by Devaney [D89]. In GW93 the authors suggested to base the definition of chaotic behavior on the notion of positive topological entropy. More recently it was shown that both Devaney chaos [HY02, and positive entropy BGKM02 imply Li-Yorke chaos. We remark that weak mixing as well (or even scattering) implies Li-Yorke chaos. Thus, in a certain sense Li-Yorke chaos is the weakest notion of Chaos. We refer the reader to the recent monograph [AAG08] and the review GY08 on local entropy theory, which include discussions of the above notions.

It is natural to ask which transitive systems are chaotic and this is the main theme of this work. In Section 1 we introduce our terminology and review some basic facts. In Section 2 we first prove, the somewhat surprising fact (Theorem 2.6) that every transitive system is partially rigid. This is then used in Section 3 to deduce the following criterion. For a transitive topological dynamical system $(X, T)$ if there is a subsystem $(Y, T)$ of $(X, T)$ (i.e. $Y$ is a non-empty closed and $T$-invariant subset of $X)$ such that $(X \times Y, T \times T)$ is transitive, then $(X, T)$ is strongly Li-Yorke chaotic. As we will see many of the known sufficient conditions in the literature, as well as a few new results, are corollaries of this fact. In fact the kind of chaotic behavior we deduce in these results is a much stronger variant of Li-Yorke chaos which we call uniform chaos. In Section 4 we reexamine these results in view of the Kuratowski-Mycielski theory. In Section 5 we specialize to minimal dynamical systems. After reviewing some structure theory we show, among other results that for minimal systems uniform chaos is preserved by extensions, and that if a minimal system is not uniformly chaotic then it is a PI system. We also show that a minimal strictly PI system which is not point distal admits a proximal scrambled Mycielski set. This perhaps suggests that a minimal system which does not contain such a set is actually point distal, but we have to leave that issue as an open problem.

Throughout the paper and mostly in Section 5 we make heavy use of enveloping semigroups and structure theory. We refer, for example, to the sources [G76], [V77], [Au88, and [Ak97] for the necessary background. 
Eli Glasner thanks his coauthors and gracious hosts, Xiangdong Ye and Song Shao for their hospitality during a long visit to Hefei in 2004, where most of this work was done.

\section{Preliminary definitions and Results}

In this section we briefly review some basic definitions and results from topological dynamics. Relevant references are [GM89, GW93, [AAB96, Ak97, AG01, [HY02, Ak03, AAG08. The latter is perhaps a good starting point for a beginner. One can also try to trace the historical development of these notions from that source and the reference list thereof.

1.1. Transitivity and related notions. We write $\mathbb{Z}$ to denote the integers, $\mathbb{Z}_{+}$ for the non-negative integers and $\mathbb{N}$ for the natural numbers. Throughout this paper a topological dynamical system (TDS for short) is a pair $(X, T)$, where $X$ is a nonvacuous compact metric space with a metric $d$ and $T$ is a continuous surjective map from $X$ to itself. A closed invariant subset $Y \subset X$ defines naturally a subsystem $(Y, T)$ of $(X, T)$.

For subsets $A, B \subset X$ we define for a TDS $(X, T)$ the hitting time set $N(A, B):=$ $\left\{n \in \mathbb{Z}_{+}: A \cap T^{-n} B \neq \emptyset\right\}$. When $A=\{x\}$ is a singleton we write $\operatorname{simply} N(x, B)$ and if moreover $B$ is a neighborhood of $x$ we refer to $N(x, B)$ as the set of return times.

Recall that $(X, T)$ is called topologically transitive (or just transitive) if for every pair of nonempty open subsets $U$ and $V$, the set $N(U, V)$ is non-empty.

Let $\omega(x, T)$ be the set of the limit points of the orbit of $x$,

$$
\operatorname{Orb}(x, T):=\left\{x, T(x), T^{2}(x), \ldots\right\} .
$$

A point $x \in X$ is called a transitive point if $\omega(x, T)=X$. It is easy to see that if $(X, T)$ is transitive then the set of all transitive points is a dense $G_{\delta}$ set of $X$ (denoted by $X_{t r}$ or Trans $\left.(X)\right)$. If $X_{t r}=X$ then we say that $(X, T)$ is minimal. Equivalently, $(X, T)$ is minimal if and only if it contains no proper subsystems. It is well known that there is some minimal subsystem in any dynamical system $(X, T)$, which is called a minimal set of $X$. Each point belonging to some minimal set of $X$ is called a minimal point.

$\mathrm{A} \operatorname{TDS}(X, T)$ is (topologically) weakly mixing if the product system $(X \times X, T \times T)$ is transitive.

A pair $(x, y) \in X \times X$ is said to be proximal if $\liminf _{n \rightarrow+\infty} d\left(T^{n} x, T^{n} y\right)=0$ and it is called asymptotic when $\lim _{n \rightarrow+\infty} d\left(T^{n} x, T^{n} y\right)=0$. If in addition $x \neq y$, then $(x, y)$ is a proper proximal (or asymptotic) pair. The sets of proximal pairs and asymptotic pairs of $(X, T)$ are denoted by $P(X, T)$ and $A \operatorname{sym}(X, T)$ respectively. A point $x \in X$ is a recurrent point if there are $n_{i} \nearrow+\infty$ such that $T^{n_{i}} x \rightarrow x$. A pair $(x, y) \in X^{2}$ which is not proximal is said to be distal. A pair is said to be a $L i$-Yorke pair if it is proximal but not asymptotic. A pair $(x, y) \in X^{2} \backslash \Delta_{X}$ is said to be a strong $L i$-Yorke pair if it is proximal and is also a recurrent point of $X^{2}$. Clearly a strong Li-Yorke pair is a Li-Yorke pair. A system without proper proximal pairs (Li-Yorke pairs, strong Li-Yorke pairs) is called distal (almost distal, 
semi-distal respectively). It follows directly from the definitions that a distal system is almost distal and an almost distal system is semi-distal. A point $x$ is called a distal point if its proximal cell $P[x]=\left\{x^{\prime} \in X:\left(x, x^{\prime}\right) \in P(X, T)\right\}=\{x\}$. A system $(X, T)$ is point distal if it contains a distal point. A theorem of Ellis [E73] says that in a metric minimal point distal system the set of distal points is dense and $G_{\delta}$. A dynamical system $(X, T)$ is equicontinuous if for every $\epsilon>0$ there is $\delta>0$ such that $d(x, y)<\delta$ implies $d\left(T^{n} x, T^{n} y\right)<\epsilon$, for every $n \in \mathbb{Z}_{+}$. Clearly an equicontinuous system is distal.

A homomorphism (or a factor map) $\pi:(X, T) \longrightarrow(Y, S)$ is a continuous onto map from $X$ to $Y$ such that $S \circ \pi=\pi \circ T$. In this situation $(X, T)$ is said to be an extension of $(Y, S)$ and $(Y, S)$ is called a factor of $(X, T)$. A homomorphism $\pi$ is determined by the corresponding closed invariant equivalence relation $R_{\pi}=$ $\left\{\left(x_{1}, x_{2}\right): \pi x_{1}=\pi x_{2}\right\}=(\pi \times \pi)^{-1} \Delta_{Y} \subset X \times X$.

An extension $\pi:(X, T) \rightarrow(Y, S)$ is called asymptotic if $R_{\pi} \subset \operatorname{Asmp}(X, T)$. Similarly we define proximal, distal extensions. We define $\pi$ to be an equicontiuous extension if for every $\epsilon>0$ there is $\delta>0$ such that $(x, y) \in R_{\pi}$ and $d(x, y)<\delta$ implies $d\left(T^{n} x, T^{n} y\right)<\epsilon$, for every $n \in \mathbb{Z}_{+}$. The extension $\pi$ is called almost one-toone if the set $X_{0}=\left\{x \in X: \pi^{-1}(\pi(x))=\{x\}\right\}$ is a dense $G_{\delta}$ subset of $X$.

1.2. The enveloping semigroup. An Ellis semigroup is a semigroup equipped with a compact Hausdorff topology such that for every $p \in E$ the map $R_{p}: E \rightarrow E$ defined by $R_{p}(q)=q p$ is continuous. (This is sometimes called a right topological, or a left topological, or a right semi-topological semigroup. Here we try to use a non-ambiguous term which we hope will standardize the terminology.) An Ellis action is an action of an Ellis semigroup $E$ on a compact Hausdorff space $X$ such that for every $x \in X$ the map $R_{x}: E \rightarrow X$ defined by $R_{x}(q)=q x$ is continuous.

The enveloping semigroup $E=E(X, T)=E(X)$ of a dynamical system $(X, T)$ is defined as the closure in $X^{X}$ (with its compact, usually non-metrizable, pointwise convergence topology) of the set $\left\{T^{n}: n \in \mathbb{Z}_{+}\right\}$. With the operation of composition of maps this is an Ellis semigroup and the operation of evaluation is an Ellis action of $E(X, T)$ on $X$ which extends the action of $\mathbb{Z}_{+}$via $T$.

The elements of $E(X, T)$ may behave very badly as maps of $X$ into itself; usually they are not even Borel measurable. However our main interest in the enveloping semigroup lies in its algebraic structure and its dynamical significance. A key lemma in the study of this algebraic structure is the following:

Lemma 1.1 (Ellis). If $E$ is an Ellis semigroup, then $E$ contains an idempotent; i.e., an element $v$ with $v^{2}=v$.

In the next proposition we state some basic properties of the enveloping semigroup $E=E(X, T)$.

Proposition 1.2. (1) A subset I of $E$ is a minimal left ideal of the semigroup $E$ if and only if it is a minimal subsystem of $(E, T)$. In particular a minimal left ideal is closed. We will refer to it simply as a minimal ideal. Minimal ideals $I$ in $E$ exist and for each such ideal the set of idempotents in I, denoted by $J=J(I)$, is non-empty. 
(2) Let I be a minimal ideal and $J$ its set of idempotents then:

(a) For $v \in J$ and $p \in I, p v=p$.

(b) For each $v \in J, v I=\{v p: p \in I\}=\{p \in I: v p=p\}$ is a subgroup of $I$ with identity element $v$. For every $w \in J$ the map $p \mapsto w p$ is a group isomorphism of $v I$ onto $w I$.

(c) $\{v I: v \in J\}$ is a partition of $I$. Thus if $p \in I$ then there exists a unique $v \in J$ such that $p \in v I$.

(3) Let $K, L$, and $I$ be minimal ideals of $E$. Let $v$ be an idempotent in $I$, then there exists a unique idempotent $v^{\prime}$ in $L$ such that $v v^{\prime}=v^{\prime}$ and $v^{\prime} v=v$. (We write $v \sim v^{\prime}$ and say that $v^{\prime}$ is equivalent to $v$.) If $v^{\prime \prime} \in K$ is equivalent to $v^{\prime}$, then $v^{\prime \prime} \sim v$. The map $p \mapsto p v^{\prime}$ of $I$ to $L$ is an isomorphism of dynamical systems.

(4) A pair $\left(x, x^{\prime}\right) \in X \times X$ is proximal if and only if $p x=p x^{\prime}$ for some $p \in E$, if and only if there exists a minimal ideal $I$ in $E$ with $p x=p x^{\prime}$ for every $p \in I$.

(5) If $(X, T)$ is minimal, then the proximal cell of $x$

$$
P[x]=\left\{x^{\prime} \in X:\left(x, x^{\prime}\right) \in P\right\}=\{v x: v \in \hat{J}\},
$$

where $\hat{J}=\bigcup\{J(I): I$ is a minimal left ideal in $E(X, T)\}$ is the set of minimal idempotents.

We will make use also of the adherence semigroup $A(X, T)$ which is defined as the $\omega$-limit set of the collection $\left\{T^{n}: n \in \mathbb{Z}_{+}\right\}$in $E(X, T)$.

Often one has to deal with more than one system at a time; e.g., we can be working simultaneously with a system and its factors, two different systems, their product, subsystems of the product, etc. Or, given a topological system $(X, T)$ we may have to work with associated systems like the action induced on the space $C(X)$ of closed subsets of $X$, with its Hausdorff topology. It is therefore convenient to have one enveloping semigroup acting on all of the systems simultaneously. This can be easily done by considering the enveloping semigroup of the product of all the systems under consideration. However, one looses nothing and gains much in convenience as well as in added machinery if one works instead with the "universal" enveloping semigroup.

Such a universal object for $\mathbb{Z}$-actions is $\beta \mathbb{Z}$, the Cech-Stone compactification of the integers (and $\beta \mathbb{Z}_{+}$for $\mathbb{Z}_{+}$-actions). These are Ellis semigroups and any $\mathbb{Z}$ (or $\mathbb{Z}_{+}$) action on $X$ via $T$ extends naturally to an Ellis action of $\beta \mathbb{Z}$ (resp. $\beta \mathbb{Z}_{+}$) on $X$.

We will freely use this fact and thus will let $\beta \mathbb{Z}_{+}$"act" on every compact $\mathbb{Z}_{+}$ dynamical system. In this case the corona $\beta^{*} \mathbb{Z}_{+}=\beta \mathbb{Z}_{+} \backslash \mathbb{Z}_{+}$coincides with the adherence semigroup. We refer to [G76, Au88, Ak97] and G03 for more details.

1.3. Some notions of Chaos. A subset $A \subset X$ is called scrambled (strongly scrambled) if every pair of distinct points in $A$ is Li-Yorke (strong Li-Yorke). The system $(X, T)$ is said to be Li-Yorke chaotic (strong Li-Yorke chaotic) if it contains an uncountable scrambled (strongly scrambled) set. 
The notion of equicontinuity can be localized in an obvious way. Namely, $x \in$ $X$ is called an equicontinuity point if for every $\epsilon>0$ there is $\delta>0$ such that $d(x, y)<\delta$ implies $d\left(T^{n} x, T^{n} y\right)<\epsilon$ for all $n \in \mathbb{Z}_{+}$. A transitive TDS is called almost equicontinuous if it has at least one equicontinuity point. If a transitive system is almost equicontinuous then the set of equicontinuity points coincides with the set of transitive points and hence it is dense $G_{\delta}$. A transitive $\operatorname{TDS}(X, T)$ is called sensitive if there is an $\epsilon>0$ such that whenever $U$ is a nonempty open set there exist $x, y \in U$ such that $d\left(T^{n} x, T^{n} y\right)>\epsilon$ for some $n \in \mathbb{N}$. A transitive TDS is either almost equicontinuous or sensitive. In particular a minimal system is either equicontinuous or sensitive (see [GW93] and [AAB96]).

A TDS $(X, T)$ is said to be chaotic in the sense of Devaney (or an infinite $P$ system) if it is transitive and $X$ is infinite with a dense set of periodic points. Such a system is always sensitive (see [BBCDS92] and GW93]).

1.4. Families and filters. We say that a collection $\mathcal{F}$ of subsets of $\mathbb{Z}_{+}$(or $\mathbb{Z}$ ) is a a family if it is hereditary upward, i.e. $F_{1} \subseteq F_{2}$ and $F_{1} \in \mathcal{F}$ imply $F_{2} \in \mathcal{F}$. A family $\mathcal{F}$ is called proper if it is neither empty nor the entire power set of $\mathbb{Z}_{+}$, or, equivalently if $\mathbb{Z}_{+} \in \mathcal{F}$ and $\emptyset \notin \mathcal{F}$. If a family $\mathcal{F}$ is closed under finite intersections and is proper, then it is called a filter. A collection of nonempty subsets $\mathcal{B}$ is a filter base if for every $B_{1}, B_{2} \in \mathcal{B}$ there is $B_{3} \in \mathcal{B}$ with $B_{3} \subset B_{1} \cap B_{2}$. When $\mathcal{B}$ is a filter base then the family

$$
\mathcal{F}=\{F: \exists B \in \mathcal{B} \text { with } B \subset F\},
$$

is a filter. A maximal filter is called an ultrafilter. By Zorn's lemma every filter is contained in an ultrafilter.

For a family $\mathcal{F}$ its dual is the family $\mathcal{F}^{*}:=\left\{F \subseteq \mathbb{Z}_{+} \mid F \cap F^{\prime} \neq \emptyset\right.$ for all $\left.F^{\prime} \in \mathcal{F}\right\}$. Any nonempty collection $\mathcal{A}$ of subsets of $\mathbb{Z}_{+}$generates a family $\mathcal{F}(\mathcal{A}):=\left\{F \subseteq \mathbb{Z}_{+}\right.$: $F \supset A$ for some $A \in \mathcal{A}\}$.

The collection $\beta \mathbb{Z}$ of ultrafilters on $\mathbb{Z}$ can be identified with the Čech-Stone compactification of the integers, where to $n \in \mathbb{Z}$ corresponds the principle unltrafilter $\{A: n \in A \subset \mathbb{Z}\}$. Using the universal property of this compactification one shows that the map $n \mapsto n+1$ on $\mathbb{Z}$ extends to a homeomorphism $S: \beta \mathbb{Z} \rightarrow \beta \mathbb{Z}$ and that, more generally, addition in $\mathbb{Z}$ can be extended to a binary operation on $\beta \mathbb{Z}$ making it an Ellis semigroup; i.e. for every $p \in \beta \mathbb{Z}$, right multiplication $R_{p}: q \mapsto q p$ is continuous. In fact the resulting dynamical system $(\beta \mathbb{Z}, S)$ is the universal point transitive dynamical system and the corresponding enveloping semigroup is naturally identified with $\beta \mathbb{Z}$ itself via the map $p \mapsto L_{p}$, where $L_{p}: q \mapsto p q$. A similar construction defines $\beta \mathbb{Z}_{+}$. In view of these facts the Ellis semigroup $\beta \mathbb{Z}$ (or $\beta \mathbb{Z}_{+}$) can serve as a universal enveloping semigroup (see Subsection 1.2 above).

Lemma 1.3. Let $(X, T)$ be a transitive TDS. Then the collection of sets

$$
\mathcal{A}=\{N(U, U): U \text { is a nonempty open subset of } X\}
$$

is a filter base, whence the family $\mathcal{F}(\mathcal{A})$ is a filter. 
Proof. Let $U_{1}$ and $U_{2}$ be nonempty open subsets of $X$. As $(X, T)$ is transitive, there is an $n \in \mathbb{N}$ such that $U_{3}=U_{1} \cap T^{-n} U_{2} \neq \emptyset$. Then

$$
\begin{aligned}
N\left(U_{3}, U_{3}\right) & \subseteq N\left(U_{1}, U_{1}\right) \cap N\left(T^{-n} U_{2}, T^{-n} U_{2}\right) \\
& =N\left(U_{1}, U_{1}\right) \cap N\left(T^{n} T^{-n} U_{2}, U_{2}\right) \\
& \subseteq N\left(U_{1}, U_{1}\right) \cap N\left(U_{2}, U_{2}\right),
\end{aligned}
$$

and our claim follows.

For a $\operatorname{TDS}(X, T)$ and a point $x \in X$ define

$$
\mathcal{I}_{x}=\{N(x, U): U \text { is a neighborhood of } x\} .
$$

A point $x$ is recurrent for $(X, T)$ if and only if each such return time set $N(x, U)$ is nonempty and so if and only if $\mathcal{I}_{x}$ is a filter base. For a pair $\left(x_{1}, x_{2}\right) \in X \times X$ define

$$
\mathcal{P}_{\left(x_{1}, x_{2}\right)}=\left\{N\left(\left(x_{1}, x_{2}\right), V\right): V \text { is a neighborhood of the diagonal in } X \times X\right\} .
$$

A pair $\left(x_{1}, x_{2}\right)$ is proximal if and only if each such $N\left(\left(x_{1}, x_{2}\right), V\right)$ is nonempty and so if and only if $\mathcal{P}_{\left(x_{1}, x_{2}\right)}$ is a filter base.

\section{TRANSITIVITY, RIGIDITY AND PROXIMALITY}

2.1. Rigid and proximal sets. The following definitions are from GM89, where they were defined for the total space $X$.

Definition 2.1. Let $(X, T)$ be a TDS , $K \subseteq X$ and $S \subset \mathbb{Z}_{+}$.

(1) We say that $K$ is rigid with respect to a sequence $S=\left\{n_{k}\right\}_{k=1}^{\infty}, n_{k} \nearrow+\infty$ if $\lim _{k \rightarrow \infty} T^{n_{k}} x=x$ for every $x \in K$.

(2) $K$ is uniformly rigid with respect to $S$ if for every $\epsilon>0$ there is an $n \in S$ with $d\left(T^{n} x, x\right)<\epsilon$ for all $x$ in $K$.

(3) $K$ is weakly rigid with respect to $S$ if every finite subset of $K$ is uniformly rigid with respect to $S$.

In items (2) and (3) we omit the reference to $S$ when $S=\mathbb{Z}_{+}$. Clearly

$$
\text { uniform rigidity } \Rightarrow \text { rigidity } \Rightarrow \text { weak rigidity. }
$$

Recall that the $A(X, T)$, the adherence semigroup of $(X, T)$, is defined as the $\omega$ limit set of the collection $\left\{T^{n}: n \in \mathbb{Z}_{+}\right\}$in $E(X, T)$. We have the following lemma (see [GM89]).

Lemma 2.2. A subset $K \subset X$ is weakly rigid if and only if there is an idempotent $u \in A(X, T)$ with $u x=x$ for every $x \in K$. In particular the identity map id $: X \rightarrow$ $X$ is an element of $A(X, T)$ if and only if the system $(X, T)$ is weakly rigid.

Proof. A subset $K \subset X$ is weakly rigid if and only if $\bigcup\left\{\mathcal{I}_{x}: x \in K\right\}$ is a filter base and so is contained in some ultrafilter. This implies that when $K$ is weakly rigid the set

$$
S_{K}=\{p \in A(X, T): p x=x \text { for every } x \in K\}
$$

is a nonempty closed subsemigroup of $A(X, T)$. By Ellis' lemma there is an idempotent $u \in S_{K}$. The converse is clear. 
Remark 2.3. We let for $n \geq 1$,

$$
\operatorname{Recur}_{n}(X)=\left\{\left(x_{1}, \ldots, x_{n}\right) \in X^{n}: \forall \epsilon>0, \exists k \in \mathbb{Z}_{+} \text {with } d\left(T^{k} x_{i}, x_{i}\right)<\epsilon, \forall i\right\} .
$$

In this notation $K \subset X$ is weakly rigid if and only if for every $n$, every $n$-tuple $\left(x_{1}, \ldots, x_{n}\right) \in K^{n}$ is in $\operatorname{Recur}_{n}(X)$. Note that $\operatorname{Recur}_{n}(X)$ is a $G_{\delta}$ subset of $X^{n}$.

Definition 2.4. Let $(X, T)$ be a TDS, $K \subset X$ and $S \subset \mathbb{Z}_{+}$.

(1) A subset $K$ of $X$ is called pairwise proximal if every pair $\left(x, x^{\prime}\right) \in K \times K$ is proximal.

(2) The subset $K$ is called uniformly proximal with respect to $S$ if for every $\epsilon>0$ there is $n \in S$ with $\operatorname{diam} T^{n} K<\epsilon$.

(3) A subset $K$ of $X$ is called proximal with respect to $S$ if every finite subset of $K$ is uniformly proximal with respect to $S$.

Remark 2.5. Thus, $K$ is uniformly proximal with respect to $S$ when there is a sequence $\left\{n_{k}\right\}$ in $S$ such that diam $T^{n_{k}} K$ converges to 0 . A subset $K \subset X$ is proximal if and only if $\bigcup\left\{\mathcal{P}_{\left(x_{1}, x_{2}\right)}:\left(x_{1}, x_{2}\right) \in K \times K\right\}$ is a filter base and so is contained in some ultrafilter. It follows that $K \subset X$ is a proximal set if and only if there exists an element $p \in E(X, T)$ with $p K=\{x\}$ for some $x \in X$. We let for $n \geq 1$,

$$
\begin{aligned}
\operatorname{Prox}_{n}(X)=\{ & \left(x_{1}, x_{2}, \cdots, x_{n}\right): \forall \epsilon>0 \exists m \in \mathbb{N} \text { such that } \\
& \left.\operatorname{diam}\left(\left\{T^{m} x_{1}, \cdots, T^{m} x_{n}\right\}\right)<\epsilon\right\} .
\end{aligned}
$$

In this notation $K \subset X$ is a proximal set if and only if for every $n$, every $n$-tuple $\left(x_{1}, \ldots, x_{n}\right) \in K^{n}$ is in $\operatorname{Prox}_{n}(X)$. Again we note that $\operatorname{Prox}_{n}(X)$ is a $G_{\delta}$ subset of $X^{n}$.

2.2. Transitivity implies partial rigidity. A nonempty subset $K$ of a compact space $X$ is a Mycielski set if it is a countable union of Cantor sets. In the following theorem we show that every transitive TDS contains a dense weakly rigid Mycielski subset. While we will later derive this result, and more, from the KuratowskiMycielski Theorem, we include here a direct proof which employs an explicit construction rather than an abstract machinery (see Theorem 4.7 below).

Theorem 2.6. Let $(X, T)$ be a transitive TDS without isolated points. Then there are Cantor sets $C_{1} \subseteq C_{2} \subseteq \cdots$ such that $K=\bigcup_{i=1}^{\infty} C_{n}$ is a dense rigid subset of $X$ and for each $N \in \mathbb{N}, C_{N}$ is uniformly rigid.

If in additional, for each $n \in \mathbb{N}, \operatorname{Prox}_{n}(X)$ is dense in $X^{n}$, then we can require that for each $N \in \mathbb{N}, C_{N}$ is uniformly proximal, whence $K$ is a proximal set.

Proof. Let $Y=\left\{y_{1}, y_{2}, \ldots\right\}$ be a countable dense subset of $X$ and for each $n \geq 1$ let $Y_{n}=\left\{y_{1}, y_{2}, \ldots, y_{n}\right\}$. Let $\mathcal{F}$ be the smallest family containing the collection

$$
\{N(U, U): U \text { is a nonempty open subset of } X\} \text {. }
$$

Since $(X, T)$ is transitive, $\mathcal{F}$ is a filter by Lemma 1.3. Let $a_{0}=0$ and $V_{0,1}=X$. We have the following claim. 
Claim: For each $S \in \mathcal{F}^{*}$ there are sequences $\left\{a_{n}\right\} \subseteq \mathbb{N},\left\{k_{n}\right\} \subseteq S$, and sequences $\left\{U_{n}\right\}_{n=1}^{\infty}$ and $\left\{V_{n, 1}, V_{n, 2}, \cdots, V_{n, a_{n}}\right\}_{n=1}^{\infty}$ of nonempty open subsets of $X$ with the following properties:

(1) $2 a_{n-1} \leq a_{n} \leq 2 a_{n-1}+n$.

(2) $\operatorname{diam} V_{n, i}<\frac{1}{n}, i=1,2, \ldots, a_{n}$.

(3) The closures $\left\{\overline{V_{n, i}}\right\}_{i=1}^{a_{n}}$ are pairwise disjoint.

(4) $\overline{V_{n, 2 i-1}} \cup \overline{V_{n, 2 i}} \subset V_{n-1, i}, i=1,2, \cdots, a_{n-1}$.

(5) $Y_{n} \subset B\left(\bigcup_{i=1}^{a_{n}} V_{n, i}, \frac{1}{n}\right)$, where $B(A, \epsilon):=\{x \in X: d(x, A)<\epsilon\}$.

(6) $T^{k_{n}}\left(V_{n, 2 i-1} \cup V_{n, 2 i}\right) \subseteq V_{n-1, i}, i=1,2, \cdots, a_{n-1}$.

Proof of Claim: For $j=1$, take $a_{1}=2, k_{1}=1$, and $V_{1,1}, V_{1,2}$ any two nonempty open sets of diameter $<1$ with disjoint closures such that $y_{1} \in B\left(V_{1,1} \cup V_{1,2}, 1\right)$. Suppose now that for $1 \leq j \leq n-1$ we have $\left\{a_{j}\right\}_{j=1}^{n-1},\left\{k_{j}\right\}_{j=1}^{n-1}$ and $\left\{V_{j, 1}, V_{j, 2}, \cdots, V_{j, a_{j}}\right\}$, satisfying conditions (1)-(6).

Choose $2 a_{n-1} \leq a_{n} \leq 2 a_{n-1}+n$ and nonempty open subsets $V_{n, 1}^{(0)}, V_{n, 2}^{(0)}, \cdots, V_{n, a_{n}}^{(0)}$ of $X$ such that:

(a) $\operatorname{diam} V_{n, i}^{(0)}<\frac{1}{2 n}, i=1,2, \cdots, a_{n}$.

(b) The closures $\left\{\overline{V_{n, i}^{(0)}}\right\}_{i=1}^{a_{n}}$ are pairwise disjoint.

(c) $\overline{V_{n, 2 i-1}^{(0)}} \cup \overline{V_{n, 2 i}^{(0)}} \subset V_{n-1, i}, i=1,2, \cdots, a_{n-1}$.

(d) $Y_{n} \subset B\left(\bigcup_{i=1}^{a_{n}} V_{n, i}^{(0)}, \frac{1}{2 n}\right)$.

As $N\left(V_{n, i}^{(0)}, V_{n, i}^{(0)}\right) \in \mathcal{F}$ for each $1 \leq i \leq a_{n}, \bigcap_{i=1}^{a_{n}} N\left(V_{n, i}^{(0)}, V_{n, i}^{(0)}\right) \in \mathcal{F}$. Take $k_{n} \in$ $S \cap \bigcap_{i=1}^{a_{n}} N\left(V_{n, i}^{(0)}, V_{n, i}^{(0)}\right)$. Hence there are nonempty open sets $V_{n, i}^{(1)} \subseteq V_{n, i}^{(0)}, 1 \leq i \leq a_{n}$, such that

(e) $T^{k_{n}}\left(V_{n, 2 i-1}^{(1)} \cup V_{n, 2 i}^{(1)}\right) \subseteq V_{n-1, i}, i=1,2, \cdots, a_{n-1}$.

Let $V_{n, i}=V_{n, i}^{(1)}, 1 \leq i \leq a_{n}$. Then the conditions (1)-(6) hold for $n$. By induction we have the claim.

Let $C_{n}=\bigcap_{j=n}^{\infty} \bigcup_{i=1}^{2^{j-n} a_{n}} \overline{V_{j, i}}$. Then $C_{1} \subseteq C_{2} \subseteq \cdots$, and by $(1)-(4), C_{n}$ is a Cantor set. By (2),(4) and (5), $K=\bigcup_{n=1}^{\infty} C_{n}$ is dense in $X$. For each $N \in \mathbb{N}$, by $(6), C_{N}$ is uniformly rigid with respect to a subsequence of $S$.

Finally, if in addition for each $n \in \mathbb{N}, \operatorname{Prox}_{n}(X)$ is dense in $X^{(n)}$ then we can in the above construction, when choosing the subsets $V_{n, i}^{(1)} \subseteq V_{n, i}^{(0)}, 1 \leq i \leq a_{n}$, add the following condition to the claim above:

(7) for each $n \in \mathbb{N}$ there is $t_{n} \in \mathbb{N}$ such that $\operatorname{diam} T^{t_{n}}\left(\bigcup_{i=1}^{a_{n}} \overline{V_{n, i}}\right)<\frac{1}{n}$.

By the requirement (7) we obtain that for each $N \in \mathbb{N}, C_{N}$ is uniformly proximal with respect to $\left\{t_{n}\right\}$. 
Remark 2.7. Using the fact that the set $X_{t r}$ of transitive points is dense in $X$ we can in the above construction, when choosing the subsets $V_{n, i}^{(1)} \subseteq V_{n, i}^{(0)}, 1 \leq i \leq a_{n}$, add the following condition to the claim in the proof:

$$
\text { (8) } Y_{n} \subseteq B\left(O r b(x, T), \frac{1}{n}\right) \text { for each } x \in \bigcup_{i=1}^{a_{n}} \overline{V_{n, i}} \text {. }
$$

It then follows that every point in $\bigcup_{i=1}^{\infty} C_{n}$ is a transitive point.

Motivated by Theorem 2.6 we define uniformly chaotic set as follows:

Definition 2.8. Let $(X, T)$ be a TDS. A subset $K \subseteq X$ is called a uniformly chaotic set if there are Cantor sets $C_{1} \subseteq C_{2} \subseteq \cdots$ such that

(1) $K=\bigcup_{i=1}^{\infty} C_{n}$ is a rigid subset of $X$ and also a proximal subset of $X$;

(2) for each $N \in \mathbb{N}, C_{N}$ is uniformly rigid; and

(3) for each $N \in \mathbb{N}, C_{N}$ is uniformly proximal.

$(X, T)$ is called (densely) uniformly chaotic, if $(X, T)$ has a (dense) uniformly chaotic subset.

Remark 2.9. Actually the fact that $K$ is rigid and proximal follows from the conditions (2) and (3): Let $J_{N}=\left\{n: d\left(T^{n} x, x\right)<1 / N\right.$ for all $\left.x \in \bigcup_{i=1}^{N} C_{i}\right\}$. Each $J_{N}$ is nonempty by assumption and $J_{N+1} \subset J_{N}$. Choose $n_{N} \in J_{N}$. As $N \rightarrow \infty$, $T^{n_{N}} x \rightarrow x$ for all $x \in \bigcup_{i=1}^{\infty} C_{i}$. Thus $K$ is a rigid subset with respect to the sequence $\left\{n_{N}\right\}$. Clearly condition (3) implies that $K$ is a proximal set.

Obviously, a uniformly chaotic set is an uncountable strongly scrambled set, hence every uniformly chaotic system is strongly Li-Yorke chaotic. Restating Theorem 2.6 we have:

Theorem 2.10. Let $(X, T)$ be a transitive TDS without isolated points. If for each $n \in \mathbb{N}, \operatorname{Prox}_{n}(X)$ is dense in $X^{(n)}$, then $(X, T)$ is densely uniformly chaotic. In particular every such system is strongly Li-York chaotic.

\section{A CRITERION FOR CHAOS AND APPLICATIONS}

\subsection{A criterion for chaos.}

Theorem 3.1 (A criterion for chaos). Let $(X, T)$ be a transitive TDS without isolated points. If there is some subsystem $(Y, T)$ of $(X, T)$ such that $(X \times Y, T)$ is transitive, then $(X, T)$ is densely uniformly chaotic.

Proof. By Theorem 2.10, it suffices to show that for each $n \in \mathbb{N}, \operatorname{Prox}_{n}(X)$ is dense in $X^{(n)}$. For a fixed $n \in \mathbb{N}$ and any $\epsilon>0$ let

$$
P_{n}(\epsilon)=\left\{\left(x_{1}, x_{2}, \ldots, x_{n}\right): \exists m \in \mathbb{N} \text { such that } \operatorname{diam}\left(\left\{T^{m} x_{1}, \ldots, T^{m} x_{n}\right\}\right)<\epsilon\right\} .
$$

Thus $\operatorname{Prox}_{n}(X)=\bigcap_{m=1}^{\infty} P_{n}\left(\frac{1}{m}\right)$ and by Baire's category theorem it is enough to show that for every $\epsilon>0, P_{n}(\epsilon)$ is a dense open subset of $X^{n}$. 
Fix $\epsilon>0$, let $U_{1}, U_{2}, \cdots, U_{n}$ be a sequence of nonempty open subsets of $X$, and let $W$ be a nonempty open subset of $Y$ with $\operatorname{diam}(W)<\epsilon$. By assumption $(X \times Y, T)$ is transitive, whence

$$
N\left(U_{1} \times W, U_{2} \times W\right)=N\left(U_{1}, U_{2}\right) \cap N(W \cap Y, W \cap Y) \neq \emptyset .
$$

Let $m_{2}$ be a member of this intersection. Then

$$
U_{1} \cap T^{-m_{2}} U_{2} \neq \emptyset \quad \text { and } \quad W \cap T^{-n_{2}} W \cap Y \neq \emptyset .
$$

By induction, we choose natural numbers $m_{3}, m_{4}, \cdots, m_{n}$ such that

$$
U_{1} \cap \bigcap_{i=2}^{n} T^{-m_{i}} U_{i} \neq \emptyset \text { and } W \cap \bigcap_{i=2}^{n} T^{-m_{i}} W \cap Y \neq \emptyset .
$$

Since $(X, T)$ is transitive, there is a transitive point $x \in U_{1} \cap \bigcap_{i=2}^{n} T^{-m_{i}} U_{i}$ and let $y \in W \cap \bigcap_{i=2}^{n} T^{-m_{i}} W$. Since $x$ is a transitive point, there exists a sequence $l_{k}$ such that $\lim _{k \rightarrow \infty} T^{l_{k}} x=y$. Thus, $\lim _{k \rightarrow \infty} T^{l_{k}}\left(T^{m_{i}} x\right)=T^{m_{i}} y$ for each $2 \leq i \leq n$. Since $\left\{y, T^{m_{2}} y, \ldots, T^{m_{3}} y\right\} \subset W$ and $\operatorname{diam}(W)<\epsilon$, for large enough $l_{k}$, we have

$$
\operatorname{diam}\left(\left\{T^{l_{k}} x, T^{l_{k}}\left(T^{m_{2}} x\right), \ldots, T^{l_{k}}\left(T^{m_{n}} x\right)\right\}\right)<\epsilon .
$$

That is, $\left(x, T^{m_{2}} x, \ldots, T^{m_{n}} x\right) \in P_{n}(\epsilon)$. Noting that $\left(x, T^{m_{2}} x, \ldots, T^{m_{n}} x\right) \in U_{1} \times U_{2} \times$ $\cdots \times U_{n}$, we have shown that

$$
P_{n}(\epsilon) \cap U_{1} \times U_{2} \times \cdots \times U_{n} \neq \emptyset
$$

As $U_{1}, U_{2}, \cdots, U_{n}$ are arbitrary, $P_{n}(\epsilon)$ is indeed dense in $X^{(n)}$.

3.2. Some applications. In the rest of this section we will obtain some applications of the above criterion. First, we need to recall some definitions (see [BHM02, HY02]).

Two topological dynamical systems are said to be weakly disjoint if their product is transitive. Call a TDS $(X, T)$ :

- scattering if it is weakly disjoint from every minimal system;

- weakly scattering if it is weakly disjoint from every minimal equicontinuous system;

- totally transitive if it is weakly disjoint from every periodic system. (Check that this is equivalent to the usual definition which requires that $\left(X, T^{n}\right)$ be transitive for all $n \geq 1$.)

Using this terminology and applying Theorem 2.10 we easily obtain the following:

Corollary 3.2. If $(X, T)$ is a TDS without isolated points and one of the following properties, then it is densely uniformly chaotic:

(1) $(X, T)$ is transitive and has a fixed point;

(2) $(X, T)$ is totally transitive with a periodic point;

(3) $(X, T)$ is scattering;

(4) $(X, T)$ is weakly scattering with an equicontinuous minimal subset;

(5) $(X, T)$ is weakly mixing.

Finally 
(6) If $(X, T)$ is transitive and has a periodic point of order $d$, then there is a closed $T^{d}$-invariant subset $X_{0} \subset X$, such that $\left(X_{0}, T^{d}\right)$ is densely uniformly chaotic and $X=\bigcup_{j=0}^{d-1} T^{j} X_{0}$. In particular $(X, T)$ is uniformly chaotic.

Proof. The only claim that needs a proof is (6). Suppose $y_{0} \in X$ is a periodic point of period $d$ and let $x_{0}$ be a transitive point; so that $\overline{\operatorname{Orb}_{T}\left(x_{0}\right)}=X$. Set $\overline{O r b_{T^{d}}\left(x_{0}\right)}=X_{0}$ (this may or may not be all of $X$ ). In any case the dynamical system $\left(X_{0}, T^{d}\right)$ is transitive, and has a fixed point. Thus, by case (1), it is densely uniformly chaotic for $T^{d}$. Both uniform proximality and uniform rigidity of subsets go over to $(X, T)$, hence $(X, T)$ is uniformly chaotic. Clearly $X=\bigcup_{j=0}^{d-1} T^{j} X_{0}$.

Part (6) provides a new proof of a result of J-H. Mai [Mai04], and as in Mai's paper we have the following corollary.

Theorem 3.3. Devaney chaos implies uniform chaos.

One can strengthen Corollary $3.2(2)$ in the following way.

Definition 3.4. Let $(X, T)$ be a TDS. A point $x \in X$ is regularly almost periodic if for each neighborhood $U$ of $x$ there is some $k \in \mathbb{N}$ such that $k \mathbb{Z}_{+} \subseteq N(x, U)$. Note that such a point is in particular a minimal point (i.e. its orbit closure is minimal).

Remark 3.5. Let $(X, T)$ be a minimal system. Then $(X, T)$ contains a regularly almost periodic point if and only if it is an almost one-to-one extension of an adding machine. If in addition $(X, T)$ is a subshift then it is isomorphic to a Toeplitz system (see e.g. [MP80]).

Next we recall the following definition from [AG01].

Definition 3.6. A property of topological dynamical systems is said to be residual if it is non-vacuous and is inherited by factors, almost one-to-one lifts, and inverse limits.

It is not hard to check that being weakly disjoint from a fixed $\operatorname{TDS}(X, T)$ is a residual property (see AG01]). One can also show that the smallest class of TDS which contains the periodic orbits and is closed under inverse limits and almost one-to-one extensions is exactly the class of almost one-to-one extensions of adding machines. It now follows that a TDS is totally transitive if and only if it is weakly disjoint from every almost one-to-one extension of an adding machine.

Corollary 3.7. If $(X, T)$ is totally transitive with a regularly almost periodic point, then it is densely uniformly chaotic.

In a similar way we see that a TDS is weakly scattering if and only if it is weakly disjoint from every system which is an almost one-to-one extension of a minimal equicontinuous system (these systems are also called almost automorphic). Thus we also have a stronger version of Corollary [3.2 (4)

Corollary 3.8. If $(X, T)$ is weakly scattering and has an almost automorphic subsystem then it is densely uniformly chaotic. 
The following example shows that we can not weaken the condition "total transitivity" to "transitivity".

Example. Let $(X, T)$ be a Toeplitz system and let $\pi: X \rightarrow Z$ be the corresponding almost one-to-one factor map from $X$ onto its maximal adding machine factor. Clearly then every proximal set in $X$ is contained in a fiber $\pi^{-1}(z)$ for some $z \in$ $Z$. Suppose now that $\left|\pi^{-1}(z)\right|<\infty$ for every $z \in Z$, and that for some $z \in Z$ there are points $x, y \in \pi^{-1}(z)$ such that $(x, y)$ is a recurrent pair and therefore a strong Li-Yorke pair (one can easily construct such systems, see e.g. [W84]). Let $Y=\overline{\operatorname{Orb}((x, y), T)} \subseteq X \times X$. By assumption the point $(x, y)$ is recurrent in $X \times X$ and forms a proximal pair. Thus the system $(Y, T)$ is transitive and, as one can easily check, has $\Delta_{X}$ as its unique minimal subset. Since $(X, T)$ is an almost one to one extension of an adding machine the diagonal $\Delta_{X} \subset Y$ contains regularly almost periodic points. However $(Y, T)$ can not be Li-Yorke chaotic because our assumption implies that every proximal set in $Y$ is finite.

This example also shows the existence of a non-minimal transitive system which is not Li-Yorke chaotic.

\section{The Kuratowski-Mycielski TheOry}

Let $X$ be a compact metric space. We recall that a subset $A \subset X$ is called a Mycielski set if it is a union of countably many Cantor sets. (This definition was introduced in BGKM02]. Note that in [Ak03] a Mycielski set is required to be dense.) The notion of independent sets and the corresponding topological machinery were introduced by Marczewski [Mar61, and Mycielski [M64]. This theory was further developed by Kuratowski in [K73]. The first application to dynamics is due to Iwanik [189]. Consequently it was used as a main tool in [BGKM02, where among other results the authors showed that positive entropy implies Li-Yorke chaos. See Ak03] for a comprehensive treatment of this topic.

In this section we first review the Kuratowski-Mycielski theory, mainly as developed in Ak03, and then consider the results of Sections 2 and 3 in view of this theory.

4.1. The Kuratowski-Mycielski Theorem. We begin by citing two classical results.

Theorem 4.1 (Ulam). Let $\phi: X \rightarrow Y$ be a continuous open surjective map with $X$ and $Y$ metric compact spaces. If $R$ is a dense $G_{\delta}$ subset of $X$, then

$$
Y_{0}=\left\{y \in Y: \phi^{-1}(y) \cap R \text { is dense in } \phi^{-1}(y)\right\}
$$

is a dense $G_{\delta}$ subset of $Y$.

Theorem 4.2 (Mycielski). Let $X$ be a complete metric space with no isolated points. Let $r_{n} \nearrow \infty$ be a sequence of positive integers and for every $n$ let $R_{n}$ be a meager subset of $X^{r_{n}}$. Let $\left\{O_{i}\right\}_{i=1}^{\infty}$ be a sequence of nonempty open subsets of $X$. Then there exists a sequence of Cantor sets $C_{i} \subset O_{i}$ such that the corresponding Mycielski 
set $K=\bigcup_{i=1}^{\infty} C_{i}$ has the property that for every $n$ and every $x_{1}, x_{2}, \ldots, x_{r_{n}}$, distinct elements of $K,\left(x_{1}, x_{2}, \ldots, x_{r_{n}}\right) \notin R_{n}$.

An especially useful instance of Mycielski's theorem is obtained as follows (see [Ak03], Theorem 5.10, and AAG08, Theorem 6.32). Let $W$ be a symmetric dense $G_{\delta}$ subset of $X \times X$ containing the diagonal $\Delta_{X}$, and let $R=X \times X \backslash W$. Let $r_{n}=n$ and set

$$
R_{n}=\left\{\left(x_{1}, \ldots, x_{n}\right):\left(x_{i}, x_{j}\right) \notin W, \forall i \neq j\right\} .
$$

Theorem 4.3. Let $X$ be a perfect compact metric space and $W$ a symmetric dense $G_{\delta}$ subset of $X \times X$ containing the diagonal $\Delta_{X}$. There exists a dense Mycielski subset $K \subset X$ such that $K \times K \subset W$.

We collect some notation and results from Akin Ak03. For $X$ a compact metric space we denote by $C(X)$ the compact space of closed subsets of $X$ equipped with the Hausdorff metric. Since $\emptyset$ is an isolated point, $C^{\prime}(X)=C(X) \backslash\{\emptyset\}$ is compact as well.

We call a collection of sets $Q \subset C^{\prime}(X)$ hereditary if it is hereditary downwards, that is, $A \in Q$ implies $C^{\prime}(A) \subset Q$ and, in particular, every finite subset of $A$ is in $Q$. For a hereditary subset $Q$ we define $R_{n}(Q)=\left\{\left(x_{1}, \ldots, x_{n}\right) \in X^{n}\right.$ : $\left.\left\{x_{1}, \ldots, x_{n}\right\} \in Q\right\}=i_{n}^{-1}(Q)$ where $i_{n}: X^{n} \rightarrow C^{\prime}(X)$ is the continuous map defined by $i_{n}\left(x_{1}, \ldots, x_{n}\right)=\left\{x_{1}, \ldots, x_{n}\right\}$. In particular, if $Q$ is a $G_{\delta}$ subset of $C^{\prime}(X)$ then $R_{n}(Q)$ is a $G_{\delta}$ subset of $X^{n}$ for all $n$. Call $A$ a $\left\{R_{n}(Q)\right\}$ set if $A^{n} \subset R_{n}(Q)$ for all $n=1,2, \ldots$ or, equivalently, if every finite subset of $A$ lies in $Q$. Clearly, the union of any chain of $\left\{R_{n}(Q)\right\}$ sets is an $\left\{R_{n}(Q)\right\}$ set and so every $\left\{R_{n}(Q)\right\}$ set is contained in a maximal $\left\{R_{n}(Q)\right\}$ set.

If $D \subset X$ we define $Q(D)=\left\{A \in C^{\prime}(X): A \subset D\right\}$, for which $R_{n}=D^{n}$. If $B \subset X \times X$ is a subset which satisfies

$$
(x, y) \in B \quad \Longrightarrow \quad(y, x),(x, x) \in B
$$

then we define $Q(B)=\left\{A \in C^{\prime}(X): A \times A \subset B\right\}$, for which $R_{2}=B$ and $\left(x_{1}, \ldots, x_{n}\right) \in R_{n}$ if and only if $\left(x_{i}, x_{j}\right) \in B$ for all $i, j=1, \ldots, n$.

If $D($ or $B)$ is a $G_{\delta}$ then so is $Q(D)$ (resp. $Q(B)$ ). Because the finite sets are dense in $C^{\prime}(X)$ it follows that if $D$ is dense in $X$ (or $B$ is $G_{\delta}$ and dense in $X \times X$ ) then $Q(D)$ (resp. $Q(B)$ ) is dense in $C^{\prime}(X)$.

Examples: (1) Let $Q($ Recur $)=\left\{A \in C^{\prime}(X): A\right.$ is uniformly rigid $\}$. We denote by $\operatorname{Recur}_{n}$ the set $R_{n}(Q(R e c u r))=\left\{\left(x_{1}, \ldots, x_{n}\right)\right.$ : recurrent in $\left.X^{n}\right\}$. The $\left\{\right.$ Recur $\left._{n}\right\}$ subsets are the weakly rigid subsets. For fixed $n$ and $\epsilon$ the condition $d\left(T^{n} x, x\right)<\epsilon$

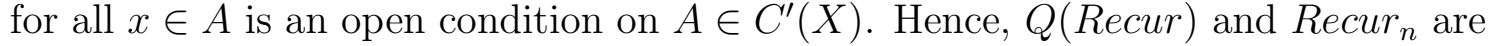
$G_{\delta}$ sets.

Notice that if $x$ is a transitive point for a transitive TDS $(X, T)$ then points of the form $\left(T^{k_{1}} x, \ldots, T^{k_{n}} x\right)$ comprise a dense set of recurrent points in $X^{n}$. Thus, for a transitive system $\operatorname{Recur}_{n}$ is dense in $X^{n}$. In addition $X_{t r}$ is a dense $G_{\delta}$ in $X$ and so $Q\left(X_{t r}\right)=\left\{A \in C^{\prime}(X): A \subset X_{t r}\right\}$ is a dense $G_{\delta}$ subset of $C^{\prime}(X)$.

(2) Let $Q(\operatorname{Prox})=\left\{A \in C^{\prime}(X): A\right.$ is uniformly proximal $\}$. We denote by $\operatorname{Prox}_{n}$ the set $R_{n}(Q(\operatorname{Prox}))$. The $\left\{\operatorname{Prox}_{n}\right\}$ subsets are the proximal subsets. For fixed 
$n$ and $\epsilon$ the condition $\operatorname{diam} T^{n} A<\epsilon$ is an open condition on $A \in C^{\prime}(X)$. Hence, $Q\left(\operatorname{Prox}_{)}\right.$and $\operatorname{Prox}_{n}$ are $G_{\delta}$ sets.

$\operatorname{Prox}_{2}=P(X, T)$ the set of proximal pairs. The $G_{\delta}$ set $Q(P(X, T))$ is the set of compacta $A$ such that $A \times A \subset P(X, T)$. The $\left\{R_{n}(Q(P))\right\}$ sets are the pairwise proximal sets.

(3) For use below we define for $Y$ a closed subset of $X$ :

$Q(T R A N S, Y)=\left\{A \in C^{\prime}(X)\right.$ : for every $\epsilon>0, n \in \mathbb{Z}_{+}$, pairwise disjoint closed $A_{1}, \ldots, A_{n} \subset A$ and $y_{1}, \ldots, y_{n} \in Y$, there exists a positive integer $k$ such that $d\left(T^{k} x, y_{i}\right)<\epsilon$ for all $\left.x \in A_{i}, i=1, \ldots, n\right\}$.

It is easy to check that $Q(T R A N S, Y)$ is a $G_{\delta}$ set, see Akin Ak03, Lemma 6.6(a). Clearly, $\left(x_{1}, \ldots, x_{n}\right) \in R_{n}(Q(T R A N S, Y))$ if and only if for every $\epsilon>0$ and $y_{1}, \ldots, y_{n} \in Y$ there exists $k$ such that $d\left(T^{k} x_{i}, y_{i}\right)<\epsilon$ for $i=1, \ldots, n$.

The point of the peculiar condition is given by

Lemma 4.4. If $K$ is a Cantor set in $X$, then $K \in Q(T R A N S, Y)$ if and only if for every continuous map $h: K \rightarrow Y$ and every $\epsilon>0$ there exists a positive integer $k$ such that $d\left(T^{k} x, h(x)\right)<\epsilon$ for all $x \in K$.

Proof. Recall that the locally constant functions on $K$, which are the continuous functions with finite range, form a dense subset of $\mathcal{C}(K, Y)$ the space of continuous functions. It thus suffices to consider such functions $h$. If $h(K)$ is the set $\left\{y_{1}, \ldots, y_{n}\right\}$ of $n$ distinct points then $\left\{A_{i}=h^{-1}\left(y_{i}\right): i=1, \ldots, n\right\}$ is a clopen partition of $K$. Hence, $K \in Q$ implies there exists a $k$ such that $T^{k} \uparrow K$ is within $\epsilon$ of $h$.

Conversely, given disjoint closed sets $A_{1}, \ldots, A_{n}$ in $K$ and points $y_{1}, \ldots, y_{n} \in Y$ there exists a clopen partition $B_{1}, \ldots, B_{n}$ of $X$ with $A_{i} \subset B_{i}$ for $i=1, \ldots, n$. The function $h: X \rightarrow Y$ with $h(x)=y_{i}$ for $x \in B_{i}$ is continuous and approximating it by some $T^{k} \uparrow K$ shows that $K \in Q$.

For a $\operatorname{TDS}(X, T)$ and closed $Y \subset X$, motivated by Lemma 4.4, we will call a Cantor set $K \in Q(T R A N S, Y)$ a Kronecker set for $Y$.

Lemma 4.5. Let $(X, T)$ be a TDS and $Y$ a closed nonempty subset of $X$. Then any Kronecker set for $Y, K \in Q(T R A N S, Y)$, is uniformly proximal. If moreover $K \subset Y$ then $K$ is also uniformly rigid, hence uniformly chaotic.

Proof. Apply Lemma 4.4. For the first assertion take $h: K \rightarrow Y$ as any constant $\operatorname{map} h: K \rightarrow Y, h(x)=y_{0}, \forall x \in K$. For the second, take $h: K \rightarrow Y$ as $h(x)=x, \forall x \in K$.

If $X$ is a perfect, nonempty, compact metric space then $C A N T O R(X)$ the set of Cantor sets in $X$ is a dense $G_{\delta}$ subset of $C^{\prime}(X)$, see e.g. Akin Ak03. Propsition $4.3(\mathrm{f})$.

The importance of all this stems from the Kuratowski-Mycielski Theorem. This version comes from Akin [Ak03] Theorem 5.10 and Corollary 5.11. 
Theorem 4.6. For $X$ a perfect, nonempty, compact metric space, let $Q$ be a $G_{\delta}$ subset of $C^{\prime}(X)$.

(a) The following conditions are equivalent

(1) For $n=1,2, \ldots, R_{n}(Q)$ is dense in $X^{n}$.

(2) There exists a dense subset $A$ of $X$ which is a $\left\{R_{n}(Q)\right\}$ set, i.e. $A^{n} \subset R_{n}(Q)$ for $n=1,2, \ldots$.

(3) $Q$ is dense in $C^{\prime}(X)$.

(4) $C A N T O R(X) \cap Q$ is a dense $G_{\delta}$ subset of $C^{\prime}(X)$.

(5) There is a sequence $\left\{K_{i}: i=1,2, \ldots\right\}$ which is dense in $C A N T O R(X)$ such that $\bigcup_{i=1}^{n} K_{i} \in Q$ for $n=1,2, \ldots$.

(b) The following conditions are equivalent

(1) There is a Cantor set in $Q$, i.e. CANTOR $(X) \cap Q \neq \emptyset$.

(2) There is a Cantor set which is an $\left\{R_{n}(Q)\right\}$ set.

(3) There is an uncountable $\left\{R_{n}(Q)\right\}$ set.

(4) There is a nonempty $\left\{R_{n}(Q)\right\}$ set with no isolated points.

(5) There is a nonempty, closed, perfect subset $Y$ of $X$ such that $Y^{n} \cap R_{n}(Q)$ is dense in $Y^{n}$ for $n=1,2, \ldots$.

4.2. Uniform chaos in light of the Kuratowski-Mycielski Theorem. With this new vocabulary we can restate Theorem 2.6 by saying that for a transitive system $(X, T)$ the collection $Q($ Recur $)$, of uniformly rigid subset, is a dense $G_{\delta}$ subset of $C^{\prime}(X)$. For the reader's convenience we repeat the statement of the theorem (augmented with a statement about pairwise proximality) and provide a short proof which employs the Kuratowski-Mycielski machinery.

Theorem 4.7. Let $(X, T)$ be a transitive TDS without isolated points. Then there are Cantor sets $C_{1} \subseteq C_{2} \subseteq \cdots$ such that $\bigcup_{i=1}^{\infty} C_{n}$ is a dense rigid subset of $X_{t r}$ and for each $N \in \mathbb{N}, C_{N}$ is uniformly rigid.

- If in addition, $P(X, T)$ is dense in $X \times X$ then we can require that $\bigcup_{i=1}^{\infty} C_{n}$ is pairwise proximal.

- If in addition, for each $n \in \mathbb{N}, \operatorname{Prox}_{n}(X)$ is dense in $X^{n}$, then we can require that for each $N \in \mathbb{N}, C_{N}$ is uniformly proximal. Thus under these conditions $(X, T)$ is uniformly chaotic.

Proof. As described in Example (1) above, Recur ${ }_{n}$ and $\left(X_{t r}\right)^{n}$ are dense $G_{\delta}$ subsets of $X^{n}$. Hence, condition (1) of part (a) of the Kuratowski-Mycielski Theorem applies to $Q($ Recur $) \cap Q\left(X_{t r}\right)$. The result follows from condition (5) of part (a) with $C_{N}=$ $\bigcup_{i=1}^{N} K_{i}$.

If $P(X, T)$ is dense in $X^{2}$ then we can intersect as well with the dense $G_{\delta}$ set $Q(P(X, T))$.

If $\operatorname{Prox}_{n}$ is dense in $X^{n}$ for every $n$ then $Q($ Prox $)$ is also a dense $G_{\delta}$ by the Kuratowski-Mycielski Theorem and so we can intersect with it as well. 
Remark 4.8. Notice that in general the collection $Q($ Recur $)$ of uniformly rigid subsets of $X$, is not finitely determined; that is, a closed subset $A \subset X$ with $A^{n} \subset$ $\operatorname{Recur}_{n}$ for every $n \geq 1$ is merely weakly rigid and need not be uniformly rigid. Similarly $Q($ Prox $)$ is not finitely determined and a closed subset $A \subset X$ with $A^{n} \subset \operatorname{Prox}_{n}$ for every $n \geq 1$ is merely a proximal set and need not be uniformly proximal.

We do likewise with the criterion for chaos (Theorem [3.1).

Theorem 4.9 (A criterion for chaos). Let $(X, T)$ be a transitive TDS without isolated points. Assume that $(Y, T)$ is a subsystem of $(X, T)$ such that $(X \times Y, T)$ is transitive, there are Cantor sets $C_{1} \subseteq C_{2} \subseteq \cdots$ such that

(1) $K=\bigcup_{i=1}^{\infty} C_{n}$ is a dense subset of $X_{t r}$ and;

(2) for each $N \in \mathbb{N}, C_{N}$ is a Kronecker set for $Y$ and is uniformly rigid.

In particular, $(X, T)$ is densely uniformly chaotic.

Proof. We follow the notation of Examples (1) and (3) above. The work below will be to show that $R_{n}(Q(T R A N S, Y))$ is dense in $X^{n}$ for $n=1,2, \ldots$ We have already seen that $\operatorname{Recur}_{n}$ is dense in $X^{n}$. By the Kuratowski-Mycielski Theorem it follows that

$$
Q(T R A N S, Y) \cap Q(\text { Recur }) \cap Q\left(X_{t r}\right)
$$

is dense in $C^{\prime}(X)$ and that the required sequence of Cantor sets exists.

Fix $\epsilon>0$ and $y_{1}, \ldots, y_{n} \in Y$ and choose open subsets $W_{1}, \ldots, W_{n}$ of diameter less than $\epsilon$ with $y_{i} \in W_{i}$ for $i=1, \ldots, n$. We will prove that the open set $\bigcup_{k \in \mathbb{Z}_{+}} T^{-k} W_{1} \times$ $\cdots \times T^{-k} W_{n}$ is dense. Then intersect over positive rational $\epsilon$ and $\left\{y_{1}, \ldots, y_{n}\right\}$ chosen from a countable dense subset of $Y$. The Baire Category Theorem then implies that $R_{n}(Q(T R A N S, Y))$ is a dense $G_{\delta}$ subset of $X^{n}$ as required.

Let $U_{1}, \ldots, U_{n}$ be open nonempty subsets of $X$. Because $X \times Y$ is transitive there exists $r_{2} \in N\left(U_{1} \times\left(W_{1} \cap Y\right), U_{2} \times\left(W_{2} \cap Y\right)\right)$. Let

$$
U_{12} \times W_{12}=\left(U_{1} \cap T^{-r_{2}} U_{2}\right) \times\left(W_{1} \cap T^{-r_{2}} W_{2}\right),
$$

an open set which meets $X \times Y$. Proceed inductively, finally choosing $r_{n} \in N\left(\left(U_{1 \ldots n-1} \times\right.\right.$ $\left.\left.\left(W_{1 \ldots n-1} \cap Y\right)\right), U_{n} \times\left(W_{n} \cap Y\right)\right)$ and let

$$
U_{1 \ldots n} \times W_{1 \ldots n}=\left(U_{1 \ldots n-1} \cap T^{-r_{n}} U_{n}\right) \times\left(W_{1 \ldots n-1} \cap T^{-r_{n}} W_{n}\right) .
$$

Choose $(x, y) \in\left(U_{1 \ldots n} \times W_{1 \ldots n}\right) \cap(Z \times Y)$ with $x \in X_{t r}$. Thus, $\left(x, T^{r_{2}} x, \ldots, T^{r_{n}} x\right) \in$ $U_{1} \times \cdots \times U_{n}$ and $\left(y, T^{r_{2}} y, \ldots, T^{r_{n}} y\right) \in W_{1} \times \cdots \times W_{n}$. Since $x$ is a transitive point, we can choose $T^{k} x$ close enough to $y$ so that $\left(T^{k} x, T^{k+r_{2}} x, \ldots, T^{k+r_{n}} x\right) \in W_{1} \times \cdots \times W_{n}$. Thus, $\left(x, T^{r_{2}} x, \ldots, T^{r_{n}} x\right) \in\left(U_{1} \times \cdots \times U_{n}\right) \cap\left(T^{-k} W_{1} \times \cdots \times T^{-k} W_{n}\right)$, as required.

For the last assertion of the theorem use Lemma 4.5. 


\section{Chaotic subsets of minimal Systems}

It is well known that a non-equicontinuous minimal system is sensitive (see GW93]). In this section we will have a closer look at chaotic behavior of minimal systems and will examine the relationship between chaos and structure theory.

5.1. On the structure of minimal systems. The structure theory of minimal systems originated in Furstenberg's seminal work [F63]. In this subsection we briefly review some of the main results of this theory. It was mainly developed for group actions and accordingly we assume for the rest of the paper that $T$ is a homeomorphism. Much of this work can be done for a general locally compact group actions, but for simplicity we stick to the traditional case of $\mathbb{Z}$-actions. We refer the reader to G76], [V77], and [Au88] for details.

We first recall that an extension $\pi: X \rightarrow Y$ of minimal systems is called a relatively incontractible (RIC) extension if it is open and for every $n \geq 1$ the minimal points are dense in the relation

$$
R_{\pi}^{n}=\left\{\left(x_{1}, \ldots, x_{n}\right) \in X^{n}: \pi\left(x_{i}\right)=\pi\left(x_{j}\right), \forall 1 \leq i \leq j \leq n\right\} .
$$

(See Theorem 7.1 in the appendix below.)

We say that a minimal system $(X, T)$ is a strictly PI system if there is an ordinal $\eta$ (which is countable when $X$ is metrizable) and a family of systems $\left\{\left(W_{\iota}, w_{\iota}\right)\right\}_{\iota \leq \eta}$ such that (i) $W_{0}$ is the trivial system, (ii) for every $\iota<\eta$ there exists a homomorphism $\phi_{\iota}: W_{\iota+1} \rightarrow W_{\iota}$ which is either proximal or equicontinuous (isometric when $X$ is metrizable), (iii) for a limit ordinal $\nu \leq \eta$ the system $W_{\nu}$ is the inverse limit of the systems $\left\{W_{\iota}\right\}_{\iota<\nu}$, and (iv) $W_{\eta}=X$. We say that $(X, T)$ is a PI-system if there exists a strictly PI system $\tilde{X}$ and a proximal homomorphism $\theta: \tilde{X} \rightarrow X$.

If in the definition of PI-systems we replace proximal extensions by almost one-toone extensions (or by highly proximal extensions in the non-metric case) we get the notion of HPI systems. If we replace the proximal extensions by trivial extensions (i.e. we do not allow proximal extensions at all) we have I systems. These notions can be easily relativize and we then speak about I, HPI, and PI extensions.

In this terminology Furstenberg's structure theorem for distal systems (Furstenberg [F63]) and the Veech-Ellis structure theorem for point distal systems (Veech [V70], and Ellis [E73]), can be stated as follows:

Theorem 5.1. A metric minimal system is distal if and only if it is an I-system.

Theorem 5.2. A metric minimal dynamical system is point distal if and only if it is an HPI-system.

Finally we have the structure theorem for minimal systems, which we will state in its relative form (Ellis-Glasner-Shapiro [EGS75], McMahon [Mc76], Veech [V77], and Glasner [G05]).

Theorem 5.3 (Structure theorem for minimal systems). Given a homomorphism $\pi: X \rightarrow Y$ of minimal dynamical system, there exists an ordinal $\eta$ (countable when 
$X$ is metrizable) and a canonically defined commutative diagram (the canonical PITower)

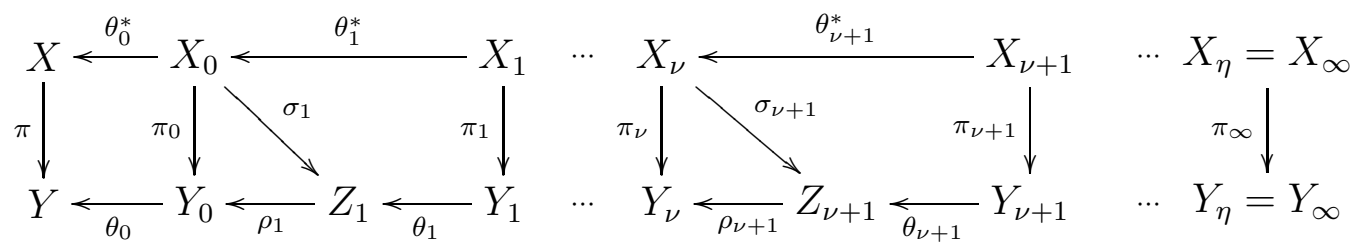

where for each $\nu \leq \eta, \pi_{\nu}$ is RIC, $\rho_{\nu}$ is isometric, $\theta_{\nu}, \theta_{\nu}^{*}$ are proximal and $\pi_{\infty}$ is $R I C$ and weakly mixing of all orders. For a limit ordinal $\nu, X_{\nu}, Y_{\nu}, \pi_{\nu}$ etc. are the inverse limits (or joins) of $X_{\iota}, Y_{\iota}, \pi_{\iota}$ etc. for $\iota<\nu$. Thus $X_{\infty}$ is a proximal extension of $X$ and a RIC weakly mixing extension of the strictly PI-system $Y_{\infty}$. The homomorphism $\pi_{\infty}$ is an isomorphism (so that $X_{\infty}=Y_{\infty}$ ) if and only if $X$ is a PI-system.

5.2. Lifting chaotic sets. Using enveloping semigroup techniques we are able to lift chaotic sets in minimal systems. First a lemma concerning proximal sets in minimal systems.

Lemma 5.4. Let $(X, T)$ be a minimal TDS and $K \subset X$ a proximal set. The set

$$
A_{K}=\left\{p \in \beta^{*} \mathbb{Z}: p K \text { is a singleton }\right\}
$$

is a closed ideal in $\beta^{*} \mathbb{Z}$. If $I \subset A_{K}$ is any minimal ideal then for any $x_{0} \in K$,

$$
K \subset\left\{v x_{0}: v \in J(I)\right\} .
$$

Proof. Because $K$ is a proximal set, $A_{K}$ is nonempty and is clearly a left ideal. Since

$$
A_{K}=\left\{p \in \beta^{*} \mathbb{Z}: p x_{1}=p x_{2} \text { for all } x_{1}, x_{2} \in K\right\}
$$

it is closed as well. Let $I \subset A_{K}$ be a minimal left ideal, which exists by Ellis' theory. Because $(X, T)$ is minimal, $I x=X$ for any $x \in K$ and so $I_{x}=\{p \in I: p x=x\}$ is a nonempty closed subsemigroup. By Ellis' Lemma there exists an idempotent $v_{x} \in I_{x}$ and since $v_{x} \in A_{K}$ and $x \in K$ we have $v_{x} K=\{x\}$. Thus, for any $x_{0} \in K$

$$
K=\left\{v_{x} x_{0}: x \in K\right\} .
$$

Remark 5.5. Because $I$ is a minimal ideal, $I=I u$ for any idempotent $u \in I$ and so $p u=p$ for any $p \in I$. In particular,

$$
v_{y} v_{x}=v_{y} \quad \text { for all } x, y \in K \text {. }
$$

Lemma 5.6. Let $\pi: X \longrightarrow Y$ be an extension between minimal systems.

(1) If $\pi$ is a proximal extension and $K \subset Y$ is a proximal set of $Y$, then any set $K^{\prime}$ of $X$ with $\pi\left(K^{\prime}\right)=K$ is a proximal set.

(2) For any proximal subset $K$ of $Y$ there is a proximal subset $K^{\prime}$ of $X$ with $\pi\left(K^{\prime}\right)=K$. 
(3) For any weakly rigid subset $K$ of $Y$, there is a weakly rigid subset $K^{\prime}$ of $X$ with $\pi\left(K^{\prime}\right)=K$. Moreover if $K$ is both proximal and weakly rigid then there is a subset $K^{\prime}$ of $X$ with $\pi\left(K^{\prime}\right)=K$ which is both proximal and weakly rigid. In particular, for any strongly Li-Yorke pair $\left(y, y^{\prime}\right)$ in $Y \times Y$ there is a strongly Li-Yorke pair $\left(x, x^{\prime}\right)$ in $X \times X$ with $\pi(x)=y, \pi\left(x^{\prime}\right)=y^{\prime}$.

(4) If $\pi$ is a distal extension and $K \subset Y$ is a weakly rigid set of $Y$, then any set $K^{\prime}$ of $X$ with $\pi\left(K^{\prime}\right)=K$ is a weakly rigid set.

In the cases (2) and (3) we have $\pi \uparrow K^{\prime}$ is one-to-one.

Proof. If $K$ is a proximal subset of $Y$ we apply Lemma 5.4 and its proof to define the ideal $A_{K}$ in $\beta^{*} \mathbb{Z}$, choose a minimal ideal $I \subset A_{K}$ and idempotents $\left\{v_{x} \in I: x \in K\right\}$ such that $v_{x} x=x$ for all $x \in K$.

1. Now assume that $\pi$ is proximal and $\pi\left(K^{\prime}\right)=K$ with $K$ a proximal subset. Let $u$ be an arbitrary idempotent in $I$ so that $u K$ is a singleton.

For any pair $x_{1}, x_{2} \in K^{\prime}$ we have

$$
\pi\left(u x_{1}\right)=u \pi\left(x_{1}\right)=u \pi\left(x_{2}\right)=\pi\left(u x_{2}\right) .
$$

As $u\left(u x_{1}, u x_{2}\right)=\left(u x_{1}, u x_{2}\right)$ and $u$ is a minimal idempotent, $\left(u x_{1}, u x_{2}\right)$ is a minimal point. Since $\pi$ is proximal, we have $u x_{1}=u x_{2}$. Since the pair $x_{1}, x_{2}$ was arbitrary, $u K^{\prime}$ is a singleton.

2. Fix $x_{0} \in X$ such that $y_{0}=\pi\left(x_{0}\right) \in K$. Assuming that $K$ is a proximal subset we define $j: K \rightarrow X$ by $j(x)=v_{x} x_{0}$. Observe that $\pi(j(x))=\pi\left(v_{x} x_{0}\right)=v_{x} y_{0}=x$. So with $K^{\prime}=j(K)$ we have $\pi\left(K^{\prime}\right)=K$. On the other hand, $y \in K$ implies $v_{y} v_{x}=v_{y}$ and so $v_{y} j(x)=v_{y} x_{0}=j(y)$ for all $x \in K$. That is, $v_{y} K^{\prime}$ is the singleton $\{j(y)\}$ and so $K^{\prime}$ is proximal.

3. Assume that $K$ is a weakly rigid subset. The set

$$
S_{K}=\left\{p \in \beta^{*} \mathbb{Z}: p y=y \text { for every } y \in K\right\}
$$

is a closed subsemigroup, nonempty because $K$ is weakly rigid. By Ellis' Lemma there is an idempotent $u \in S_{K}$. Choose for each $x \in K, j(x) \in \pi^{-1}(x)$. Let

$$
K^{\prime}=\{u j(x): x \in K\} .
$$

Since $\pi(u j(x))=u \pi(j(x))=u x=x$ it follows that $\pi\left(K^{\prime}\right)=K$. Since $u$ is an idempotent it acts as the identity on $K^{\prime}$.

Now assume in addition that $K$ is a proximal subset. $A_{K} u$ is a closed ideal. Since $u$ acts as the identity on $K$, it follows that $p u(K)$ is a singleton for every $p \in A_{K}$, i.e. $A_{K} u \subset A_{K}$. If $I$ a minimal ideal in $A_{K} u$ then with $p u=p$ for all $p \in I$. In particular, the idempotents $v_{x} \in I$ satisfy $v_{x} u=v_{x}$ and so $u v_{x} u v_{x}=u v_{x} v_{x}=u v_{x}$. That is, $u v_{x}$ is an idempotent in $I$. Furthermore, $u v_{x}(K)=\{u x\}=\{x\}$. Thus, we can replace $v_{x}$ by $u v_{x}$ if necessary and so assume that $u v_{x}=v_{x}$.

As in (2) define $j(x)=v_{x} x_{0}$ to obtain the proximal set $K^{\prime}=j(K)$. Since $u v_{x}=v_{x}$, $u j(x)=j(x)$ and so $u$ acts as the identity on $K^{\prime}$. That is, $K^{\prime}$ is a weakly rigid set as well. 
4. As in part 3. set

$$
S_{K}=\left\{p \in \beta^{*} \mathbb{Z}: p y=y \text { for every } y \in K\right\}
$$

and then pick an idempotent $u \in S_{K}$. Now for any $x \in X$ with $\pi(x) \in K$ the points $x$ and $u x$ are proximal. But as $\pi(u x)=u \pi(x)=\pi(x)$ and $\pi$ is a distal extension we conclude that $u x=x$. Thus if $\pi\left(K^{\prime}\right)=K$ then $u x=x$ for every $x \in K^{\prime}$, whence $K^{\prime}$ is weakly rigid.

Now from Theorem 4.6 it follows that a dynamical system $(X, T)$ contains a Cantor subset which is both uniformly proximal and uniformly rigid, if and only if there is an uncountable $A \subset X$ such that for every $n$-tuple $\left(x_{1}, \ldots, x_{n}\right)$ with $x_{j} \in A$ we have $\left(x_{1}, \ldots, x_{n}\right) \in \operatorname{Prox}_{n}(X) \cap \operatorname{Recur}_{n}(X)$. Thus if we let $Q(P R)=Q(\operatorname{Prox}) \cap$ $Q$ (Recur) be the collection of closed subsets of $X$ which are both uniformly proximal and uniformly rigid, then $Q(P R)$ is a $G_{\delta}$ subset of $C^{\prime}(X)$ and for every $n \geq 1$, $R_{n}(Q(P R))=\operatorname{Prox}_{n}(X) \cap \operatorname{Recur}_{n}(X)$. These facts combined with Lemma [5.6(3) yield the following important corollary.

Theorem 5.7. Let $\pi: X \rightarrow Y$ be a homomorphism of minimal systems. If $Y$ contains a uniformly chaotic subset then so does $X$.

Remark 5.8. One would like to prove analogous lifting theorems for Li-Yorke and strong Li-Yorke chaotic sets (i.e. uncountable scrambled and strongly scrambled sets). Unfortunately the collection of closed scrambled sets is not, in general, a $G_{\delta}$ subset of $C^{\prime}(X)$, and we therefore can not use this kind of argument to show that Li-Yorke chaos lifts under homomorphisms of minimal systems. The problem with lifting closed strongly scrambled sets (which do form a $G_{\delta}$ set) is that we do not know whether an uncountable strongly scrambled set can always be lifted through an extension of minimal systems.

\subsection{Weakly mixing extensions.}

Theorem 5.9. Let $(X, T)$ be a TDS and $\pi:(X, T) \rightarrow(Y, S)$ an open nontrivial weakly mixing extension. Then there is a residual subset $Y_{0} \subseteq Y$ such that for every point $y \in Y_{0}$ the set $\pi^{-1}(y)$ contains a dense strongly scrambled Mycielski subset $K$ such that $K \times K \backslash \Delta_{X} \subseteq \operatorname{Trans}\left(R_{\pi}\right)$. In particular $(X, T)$ is strongly Li-Yorke chaotic.

If moreover $\pi$ is weakly mixing and RIC, then there is a residual subset $Y_{0} \subseteq Y$ such that for every point $y \in Y_{0}$ a dense Mycielski set $K \subset \pi^{-1}(y), y \in Y_{0}$ as above can be found which is uniformly chaotic, whence $X$ is uniformly chaotic.

Proof. Since $\pi$ is open, it follows that $\pi \times \pi: R_{\pi} \rightarrow Y,\left(x_{1}, x_{2}\right) \mapsto \pi\left(x_{1}\right)$ is open as well. Since $R_{\pi}$ is transitive, the set of transitive points $\operatorname{Trans}\left(R_{\pi}\right)$ is a dense $G_{\delta}$ subset of $R_{\pi}$. By Ulam's Theorem there is a residual subset $Y_{0} \subseteq Y$ such that for every point $y \in Y_{0}$,

$$
\operatorname{Trans}\left(R_{\pi}\right) \cap \operatorname{Recur}_{2} \cap\left(\pi_{\infty}^{-1}(y) \times \pi_{\infty}^{-1}(y)\right)
$$

is dense $G_{\delta}$ in $\left(\pi^{-1}(y) \times \pi^{-1}(y)\right)$. 
Now for each $y \in Y_{0}$, we claim that $\pi^{-1}(y)$ has no isolated points. In fact if this is not true, then there exists $x \in \pi^{-1}(y)$ such that $\{x\}$ is an open subset of $\pi^{-1}(y)$. Moreover, $\{(x, x)\}$ is an open subset of $\pi^{-1}(y) \times \pi^{-1}(y)$. Since $\operatorname{Trans}\left(R_{\pi}\right) \cap\left(\pi^{-1}(y) \times\right.$ $\left.\pi^{-1}(y)\right)$ is dense $G_{\delta}$ in $\left(\pi^{-1}(y) \times \pi^{-1}(y)\right)$, one has $(x, x) \in \operatorname{Trans}\left(R_{\pi}\right)$. This shows that $R_{\pi}=\Delta_{X}$ which contradicts the fact that $\pi$ is a non-trivial extension. Finally, by Theorem 4.3 there is a dense s-chaotic subset $K \subseteq \pi^{-1}(y)$ such that

$$
K \times K \backslash \Delta_{X} \subseteq \operatorname{Trans}\left(R_{\pi}\right) \cap\left(\pi^{-1}(y) \times \pi^{-1}(y)\right) \backslash \Delta_{X} \subseteq \operatorname{Trans}\left(R_{\pi}\right) .
$$

We now further assume that $\pi$ is a RIC extension. Then by G05], Theorem 2.7, $\pi$ is weakly mixing of all orders (i.e. $R_{\pi}^{n}$ is transitive for all $n \geq 2$ ) and in particular for every $n \geq 2$,

$$
\operatorname{Prox}_{n} \cap \operatorname{Recur}_{n} \cap \pi^{-1}(y)^{n}
$$

is a dense in $\pi^{-1}(y)^{n}$, for every $y \in Y_{0}$. Applying Theorem 4.6 we obtain our claim.

5.4. The non PI case. The following theorems of Bronstein Bro79 and van der Woude [Wo85] give intrinsic characterizations of PI-extensions and HPI-extensions respectively. Recall that a map $\pi: X \rightarrow Y$ between compact spaces is called semiopen if int $\pi(U) \neq \emptyset$ for every nonempty open subset $U \subset X$. It was observed by J. Auslander and N. Markley that a homomorphism $\pi: X \rightarrow Y$ between minimal systems is always semi-open (see e.g. G05], Lemma 5.3).

Theorem 5.10. Let $\pi:(X, T) \longrightarrow(Y, T)$ be a homomorphism of compact metric minimal systems. Then

(1) The extension $\pi$ is PI if and only if it satisfies the following property: whenever $W$ is a closed invariant subset of $R_{\pi}$ which is transitive and has a dense subset of minimal points, then $W$ is minimal.

(2) The extension $\pi$ is HPI if and only if it satisfies the following property: whenever $W$ is a closed invariant subset of $R_{\pi}$ which is transitive and the restriction of the projection maps to $W$ are semi-open, then $W$ is minimal.

Next we show that a minimal system which is a non-PI extension has an s-chaotic subset.

Theorem 5.11. Let $\pi:(X, T) \longrightarrow(Y, T)$ be a homomorphism of metric minimal systems. If $\pi$ is a non-PI extension, then there is a dense subset $Y_{0} \subset Y$ such that for each $y_{0} \in Y_{0}$, there is a uniformly chaotic subset of $\pi^{-1}\left(y_{0}\right)$. In particular $(X, T)$ is strongly Li-Yorke chaotic.

Proof. Assume that $\pi:(X, T) \longrightarrow(Y, T)$ is a non-PI extension. Then by Theorem 5.3 there exist $\phi:\left(X_{\infty}, T\right) \rightarrow(X, T), \pi_{\infty}:\left(X_{\infty}, T\right) \rightarrow\left(Y_{\infty}, T\right)$ and $\eta: Y_{\infty} \longrightarrow Y$ such that $\phi$ is a proximal extension, $\pi_{\infty}$ is weakly mixing RIC extension, and $\eta$ is a PI-extension. As $\pi$ is non-PI, $\pi_{\infty}$ is non-trivial. 
Now consider the commutative diagram

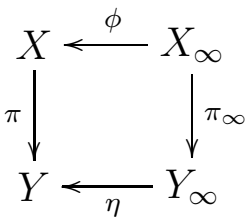

By Theorem 5.9 there is a dense $G_{\delta}$ subset $Y_{\infty}^{0} \subset Y_{\infty}$ such that, for every $y \in Y_{\infty}^{0}$, there is a dense uniformly chaotic subset $K_{y}$ of $\pi_{\infty}^{-1}(y)$.

Since $\pi$ is not PI, $\pi_{\infty}$ is not proximal. Thus, there is a distal point $\left(x_{1}, x_{2}\right) \in$ $R_{\pi_{\infty}} \backslash \Delta_{X_{\infty}}$. This implies that $\phi\left(x_{1}\right) \neq \phi\left(x_{2}\right)$ as $\phi$ is a proximal extension. For any $k_{1}, k_{2} \in K=K_{y}$ with $k_{1} \neq k_{2}$, one has $\left(k_{1}, k_{2}\right) \in \operatorname{Trans}\left(R_{\pi_{\infty}}\right)$. As $\phi\left(x_{1}\right) \neq \phi\left(x_{2}\right)$, $\left(x_{1}, x_{2}\right) \in R_{\pi_{\infty}}$ and $\left(k_{1}, k_{2}\right) \in \operatorname{Trans}\left(R_{\pi_{\infty}}\right)$, one has $\phi\left(k_{1}\right) \neq \phi\left(k_{2}\right)$. That is, $\phi$ : $K \rightarrow \phi(K)$ is a bijection. Therefore, as is easy to check, $\phi(K)$ is a uniformly chaotic subset of $X$. Moreover $\phi(K) \subset \pi^{-1}(\eta(y))$. Finally we let $Y_{0}=\eta\left(Y_{\infty}^{0}\right)$; clearly a dense subset of $Y$.

5.5. The proximal but not almost one-to-one case. Every extension of minimal systems can be lifted to an open extension by almost one-to-one modifications. To be precise, for every extension $\pi: X \rightarrow Y$ of minimal systems there exists a canonically defined commutative diagram of extensions (called the shadow diagram)

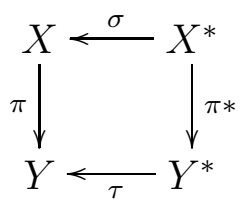

with the following properties:

(a) $\sigma$ and $\tau$ are almost one-to-one;

(b) $\pi^{*}$ is an open extension;

(c) $X^{*}$ is the unique minimal set in $R_{\pi \tau}=\left\{(x, y) \in X \times Y^{*}: \pi(x)=\tau(y)\right\}$ and $\sigma$ and $\pi^{*}$ are the restrictions to $X^{*}$ of the projections of $X \times Y^{*}$ onto $X$ and $Y^{*}$ respectively.

In [G76] it was shown that a metric minimal system $(X, T)$ with the property that $\operatorname{Prox}_{n}(X)$ is dense in $X^{n}$ for every $n \geq 2$ is weakly mixing. This was extended by van der Woude [Wo82] as follows (see also [G05]).

Theorem 5.12. Let $\pi: X \rightarrow Y$ be a factor map of the metric minimal system $(X, T)$. Suppose that $\pi$ is open and that for every $n \geq 2, \operatorname{Prox}_{n}(X) \cap R_{\pi}$ is dense in $R_{\pi}$. Then $\pi$ is a weakly mixing extension. In particular a nontrivial open proximal extension is a weakly mixing extension.

Lemma 5.13. Let $\pi: X \rightarrow Y$ be a continuous surjective map between compact metric spaces which is almost one-to-one. If $A \subset X$ is a dense $G_{\delta}$ subset, then $\pi(A)$ contains a dense $G_{\delta}$ subset of $Y$. 
Proof. Let $A_{0}=\left\{x \in X: \pi^{-1} \pi(x)=\{x\}\right\}$ and $B_{0}=\left\{y \in Y:\right.$ Card $\pi^{-1}(y)=$ $1\}$. Then $A_{0}$ (resp. $\left.B_{0}\right)$ is a dense $G_{\delta}$ subset of $X$ (resp. $Y$ ). Now $A \cap A_{0}$ is a dense $G_{\delta}$ subset of $X$, hence a dense $G_{\delta}$ of $A_{0}$. As the set of continuity points of $\pi^{-1}: Y \longrightarrow C(X)$ contains $B_{0}, \pi: A_{0} \rightarrow B_{0}$ is a homeomorphism, so $\pi\left(A \cap A_{0}\right)$ is a dense $G_{\delta}$ subset of $B_{0}$. Therefore, there exist open subsets $U_{n}$ of $Y$ such that $\bigcap_{n=1}^{\infty} U_{n} \cap B_{0}=\pi\left(A \cap A_{0}\right)$. This shows that $\pi\left(A \cap A_{0}\right)$ is also a dense $G_{\delta}$ subset of $Y$.

Recall that a subset $K$ of $X$ is a proximal set if each finite tuple from $K$ is uniformly proximal (see Definition 2.4). The proof of the following lemma is straightforward.

Lemma 5.14. Let $\pi: X \longrightarrow Y$ be a proximal extension between minimal systems. Then for each $y \in Y, \pi^{-1}(y)$ is a proximal set.

In the sequel it will be convenient to have the following:

Definition 5.15. Let $(X, T)$ be a TDS.

(1) A scrambled Mycielski subset $K \subset X$ will be called a chaotic subset of $X$.

(2) A strongly scrambled Mycielski subset will be called an s-chaotic subset of $X$.

We can now prove the following result (see also [AAG08], Theorem 6.33).

Theorem 5.16. Let $\pi: X \rightarrow Y$ be a proximal but not almost one-to-one extension between minimal systems. Then there is a residual subset $Y_{0} \subset Y$ such that for each $y \in Y_{0}, \pi^{-1}(y)$ contains a proximal s-chaotic set $K$ with $K \times K \backslash \Delta_{X} \subseteq \operatorname{Trans}\left(R_{\pi}\right)$.

Proof. In the shadow diagram for $\pi$, the map $\pi^{*}$ is open and proximal. Since $\pi$ is not almost one-to-one $\pi^{*}$ is not trivial. Thus, by Theorem 5.12, $\pi^{*}$ is a nontrivial open weakly mixing extension. Hence by Theorem 5.9 there is a residual subset $Y_{0}^{*} \subset Y^{*}$ such that for each $y^{*} \in Y_{0}^{*}, \pi^{*-1}\left(y^{*}\right)$ contains an s-chaotic set $K^{*}$ and $K^{*} \times K^{*} \backslash \Delta_{X^{*}} \subseteq \operatorname{Trans}\left(R_{\pi^{*}}\right)$. Moreover, $\pi^{*}$ being proximal, we have for every $n \geq 2, \pi^{*-1}\left(y^{*}\right)^{n} \subset \operatorname{Prox}_{n}$ and therefore we can require that $K^{*}$ be proximal as well. Since in the shadow diagram $\sigma$ and $\pi^{*}$ are the restrictions to $X^{*}$ of the projections of $X \times Y^{*}$ onto $X$ and $Y^{*}$ respectively, $\sigma\left(K^{*}\right)$ is an s-chaotic set, as $\pi \sigma\left(K^{*}\right)=\tau \pi^{*}\left(K^{*}\right)=\left\{\tau\left(y^{*}\right)\right\}, \sigma\left(K^{*}\right) \subset \pi^{-1}\left(\tau\left(y^{*}\right)\right)$. Finally, set $Y_{0}=\tau\left(Y_{0}^{*}\right)$. Since $\tau$ is almost one-to-one, $Y_{0}$ is a residual subset of $Y$ (Lemma 5.13) .

The following result was first proved in AAG08.

Corollary 5.17. Let $\pi: X \rightarrow Y$ be an asymptotic extension between minimal systems. Then $\pi$ is almost one-to-one.

Proof. We use the notations in the proof of Theorem 5.16. Note that $\pi$ is proximal. If it is not almost one-to-one, then by Theorem 5.16, there are $\sigma(x) \neq \sigma(y) \in \sigma\left(K^{*}\right)$ such that $(\sigma(x), \sigma(y))$ is a recurrent point of $T \times T$. It is clear that $\pi \sigma(x)=$ $\pi \sigma(y)$ since the diagram is commutative. It now follows that $\pi$ is not asymptotic, a contradiction. 
Remark 5.18. In GW79 the authors construct an example of a minimal system $(X, T)$ which admits a factor map $\pi: X \rightarrow Y$ such that (i) the factor $Y$ is equicontinuous, (ii) the map $\pi$ is a nontrivial open proximal extension. Now such an $X$ is clearly strictly PI but not HPI. However, according to Theorem 5.12 the extension $\pi$ is a weakly mixing extension and it follows from Theorem 5.9 that for some $y \in Y$ the fiber $\pi^{-1}(y)$ contains a dense proximal s-chaotic subset. Thus $X$ is an example of a minimal PI system which is strongly Li-Yorke chaotic. We do not have an example of a minimal PI system which contains a uniformly chaotic set.

We also note that, by BGKM02, positive topological entropy implies the existence of an s-chaotic subset. Since there are HPI systems with positive entropy (e.g many Toeplitz systems [W84]) we conclude that there are HPI systems which are strongly Li-Yorke chaotic.

5.6. The PI, non-HPI case. We have shown (Subsection 5.4) that for a non-PI extension there is a uniformly chaotic set. The natural question now is whether there is a chaotic (s-chaotic, uniformly chaotic) set for a non-HPI extension? (Recall that for a metric $X$ the notions 'HPI extension' and 'point distal extension' coincide.) At present we are unable to answer this question fully. However we will show that the answer is affirmative for a sub-class of non-HPI extensions:

Proposition 5.19. Let $\pi: X \longrightarrow Y$ be a strictly PI extension but non-HPI extension between minimal systems. Then there is a dense set $Y_{0}$ of $Y$ such that for each $y \in Y_{0}, \pi^{-1}(y)$ contains a proximal chaotic set.

Proof. Since by assumption $\pi$ is non-HPI, in its strictly PI-tower at least one of the proximal extensions in the canonical PI-tower is not an almost one-to-one extension. Let us denote this segment of the tower by

$$
X \stackrel{\pi_{1}}{\longrightarrow} Z_{1} \stackrel{\pi_{2}}{\longrightarrow} Z_{2} \stackrel{\pi_{3}}{\longrightarrow} Y
$$

with $\pi_{1} \circ \pi_{2} \circ \pi_{3}=\pi$, and where $\pi_{1}$ and $\pi_{3}$ are strictly PI extensions and $\pi_{2}$ is a proximal but not an almost one-to-one extension.

By Theorem 5.16 there exists a dense set $Z_{0} \subset Z_{2}$ such that for each $z \in Z_{2}$, $\pi_{2}^{-1}(z)$ contains a proximal s-chaotic set $K \subset Z_{1}$. By Lemma 5.6 (3), there is a proximal subset $K^{\prime} \subset X$ with $\pi_{1} \circ \pi_{1}\left(K^{\prime}\right)=K$ and as $K$ is s-scarambled, $K^{\prime}$ is at least scrambled. Now the proposition follows by setting $Y_{0}=\pi_{3}\left(Z_{0}\right)$.

Now assume that $\pi: X \longrightarrow Y$ is PI and not HPI. This means that in the canonical PI-tower there are maps $\phi: X_{\infty} \longrightarrow X$ which is proximal and $\eta: X_{\infty} \longrightarrow Y$ which is strictly PI:

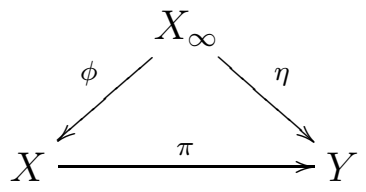

Lemma 5.20. The extension $\eta$ is not strictly HPI extension. 
Proof. Assume $\eta$ is a strictly HPI-extension. Then by Theorem 5.10. (2) there are no non-minimal transitive subsystem $W$ of $R_{\eta}$ such that the coordinate projection $W \rightarrow Y$ is semi-open. Now it is easy to see that there is no non-minimal transitive subsystem $W^{\prime}$ of $R_{\phi}$ such that the coordinate projection $W^{\prime} \rightarrow X$ is semi-open. For if $W^{\prime}$ is a non-minimal transitive subsystem of $R_{\phi}$ such that the coordinate projection $W^{\prime} \rightarrow X$ is semi-open, then $W^{\prime}$ is also a subsystem of $R_{\eta}$. But the composition of two semi-open maps is also semi-open and $\pi: X \rightarrow Y$ is semi-open, hence the coordinate projection $W^{\prime} \rightarrow Y$ is semi-open, a contradiction.

Hence using Theorem 5.10, (2) again, this shows that $\phi$ is an HPI-extension. However $\phi$ is also proximal, so we conclude that $\phi$ is almost one-to-one. This shows that $\pi$ is an HPI-extension contradicting our assumption.

Thus combining this lemma with Proposition 5.19 we know that $X_{\infty}$ contains a proximal chaotic subset $K$. However, we do not know whether its image $\phi(K) \subset X$ is also such a set.

We conclude by formally stating our open problems.

Problem 5.21. 1. A non-PI system contains a uniformly chaotic set (Theorem 5.11), is the converse true? (See remark 5.18.)

2. A strictly PI system which is not HPI contains a proximal chaotic subset (Proposition 5.19), is this true also for a PI non-HPI system? 


\section{TABLE}

In the table below we summarize the interrelations between the various kinds of chaos discussed in the paper. In each case the label refers to the existence of a large chaotic set. We write 'ch.' for chaos and 's' for strong. The labels ch. and s-ch. refer to the existence of Mycielski scrambled set and Mycielski strongly scrambled set respectively. Proximal means to say that the chaotic set in question is a proximal set.

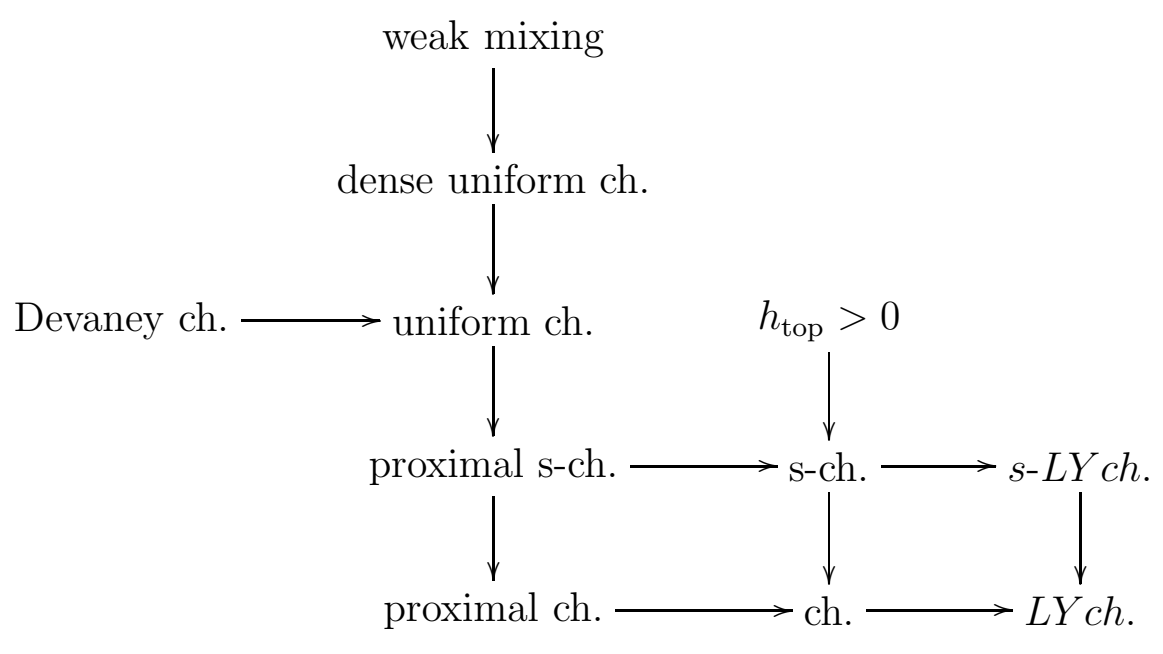

TABLE 1. Types of chaotic behavior

\section{Appendix}

7.1. A characterization of RIC extensions. Following usual notation we write $\beta \mathbb{Z}$ for the Čech-Stone compactification of the integers, and we fix a minimal left ideal $M \subset \beta \mathbb{Z}$ and an idempotent $u=u^{2} \in J(M)$, where $J(M)$ is the nonempty set of idempotents in $M$. Then the subset $G=u M$ is a maximal subgroup of the semigroup $M$ which decomposes as a disjoint union $M=\bigcup\{v G: v \in J(M)\}$. The group $G$ can be identified with the group of automorphisms of the dynamical system $(M, \mathbb{Z})$ (see e.g $\mathrm{G} 76$ or $\mathrm{Au} 88$ ). We also recall that the semigroup $\beta \mathbb{Z}$ is a universal enveloping semigroup and thus "acts" on every compact $\mathbb{Z}$ dynamical system. In particular, when $(X, T)$ is a dynamical system the homeomorphism $T$ defines in a natural way a homeomorphism on $C(X)$, the compact space of closed subsets of $X$. Now for $p \in \beta \mathbb{Z}$ the "action" of $p$ on the point $A \in C(X)$ is well defined. In order to avoid confusion here we denote the resulting element of $C(X)$ by $p \circ A$ and refer to this action as the circle operation. A more concrete definition of this set is

$$
p \circ A=\lim \sup T^{n_{i}} A,
$$

where, denoting by $S$ the generator of $\mathbb{Z}, S^{n_{i}}$ is any net in $\beta \mathbb{Z}$ which converges to $p$. Thus we always have $p A=\{p x: x \in A\} \subset p \circ A$, but usually the inclusion is proper, 
as often $p A$ is not even a closed subset of $X$. A quasifactor of a system $(X, T)$ is a closed invariant set $\mathcal{M} \subset C(X)$ such that $\bigcup\{A: A \in \mathcal{M}\}=X$.

Recall that an extension $\pi: X \rightarrow Y$ of minimal dynamical systems is called a relatively incontractible $(R I C)$ extension if for every $p \in \beta \mathbb{Z}$ we have $p \circ F x_{0}=$ $\pi^{-1}\left(p y_{0}\right)$, where $x_{0}=u x_{0}$ is a point in $X, y_{0}=\pi\left(x_{0}\right)$ and $F=\mathfrak{G}\left(Y, y_{0}\right)=\{\alpha \in G$ : $\left.\alpha y_{0}=y_{0}\right\}$ is the Ellis group of the pointed minimal system $\left(Y, y_{0}, T\right)$.

Theorem 7.1. The extension $\pi: X \rightarrow Y$ is RIC if and only if it is open and for every $n \geq 1$ the minimal points are dense in the relation

$$
R_{\pi}^{n}=\left\{\left(x_{1}, \ldots, x_{n}\right) \in X^{n}: \pi\left(x_{i}\right)=\pi\left(x_{j}\right), \forall 1 \leq i \leq j \leq n\right\} .
$$

Proof. Suppose $\pi$ is RIC. Then clearly the map $y \mapsto \pi^{-1}(y)$ is continuous, i.e. $\pi$ is an open map. Since every $n$-tuple $\left(x_{1}, \ldots, x_{n}\right)$ with $x_{i} \in F x_{0}, i=1, \ldots, n$ is a minimal point of $R_{\pi}^{n}$, the density of minimal points in $R_{\pi}^{n}$ follows from the definition of the circle operation.

Conversely, suppose $\pi$ is open and the minimal points are dense in $R_{\pi}^{n}$. We first note that the fact that $\pi$ is RIC is equivalent to the statement that the minimal quasifactor $\mathcal{M}=\left\{p \circ F x_{0}: p \in M\right\} \subset C(X)$, where $M$ is a minimal ideal in $\beta \mathbb{Z}$, coincides with the collection $\left\{\pi^{-1}(y): y \in Y\right\}$. Thus it suffices to show that for an arbitrary $y \in Y$ the point $\pi^{-1}(y)$ is in $\mathcal{M}$.

Let $d$ be any continuous semi-metric on $X$. Let $\left(x_{1}, \ldots, x_{n}\right)$ be an $n$-tuple of elements of $\pi^{-1}(y)$ which is $\epsilon$-dense in $\pi^{-1}(y)$ (with respect to $d$ ). By assumption then, there is an $n$-tuple $\left(x_{1}^{\prime}, \ldots, x_{n}^{\prime}\right)$ of elements of $\pi^{-1}\left(y^{\prime}\right)$, such that (i) $d\left(y, y^{\prime}\right)<\epsilon$, (ii) $d\left(x_{i}, x_{i}^{\prime}\right)<\epsilon$ for every $i$, and (iii) $\left(x_{1}^{\prime}, \ldots, x_{n}^{\prime}\right)$ is a minimal point of $R_{\pi}^{n}$.

There is a minimal idempotent $v \in J(M)$ such that $v x_{i}^{\prime}=x_{i}^{\prime}$ for every $i$ and it follows that $\left\{x_{1}^{\prime}, \ldots, x_{n}^{\prime}\right\} \subset v \pi^{-1}\left(y^{\prime}\right)$. Note that we must have $v y^{\prime}=y^{\prime}$ and there is therefore some $p_{y^{\prime}}=p=v p \in M$ with $y^{\prime}=p y_{0}$.

Now

$$
\left\{x_{1}^{\prime}, \ldots, x_{n}^{\prime}\right\} \subset v \pi^{-1}\left(y^{\prime}\right) \subset v \circ v \pi^{-1}\left(y^{\prime}\right)=v \circ p F x_{0}=p \circ F x_{0} \subset \pi^{-1}\left(y^{\prime}\right) .
$$

Since we have $\lim \sup _{y^{\prime} \rightarrow y} \pi^{-1}\left(y^{\prime}\right) \subset \pi^{-1}(y)$, we conclude that the set $\pi^{-1}(y)$ is a limit point of the sets $p_{y^{\prime}} \circ F x_{0} \in \mathcal{M}$. Thus also $\pi^{-1}(y) \in \mathcal{M}$ and the proof is complete.

\section{REFERENCES}

[Ak97] E. Akin, Recurrence in topological dynamical systems: Furstenberg families and Ellis actions, Plenum Press, New York, 1997.

[Ak03] E. Akin, Lectures on Cantor and Mycielski sets for dynamical systems, Chapel Hill Ergodic Theory Workshops, 21-79, Contemp. Math., 356, Amer. Math. Soc., Providence, RI, 2004.

[AAB96] E. Akin, J. Auslander and K. Berg, When is a transitive map chaotic?, Convergence in Ergodic Theory and Probability (Columbus, OH, 1993) (Ohio University Math. Res. Inst. Pub., 5). de Gruyter, Berlin, (1996), 25-40.

[AAG08] E. Akin, J. Auslander and E. Glasner, The topological dynamics of Ellis actions. Mem. Amer. Math. Soc. 195, (2008), no. 913 
[AG01] E. Akin and E. Glasner, Residual properties and almost equicontinuity, J. d'Analyse Math. 84, (2001), 243-286.

[Au88] J. Auslander, Minimal flows and their extensions, North-Holland Mathematics Studies, 153, Amsterdam, 1988.

[BBCDS92] J. Banks, J. Brooks, G. Cairns, G. Davis and P. Stacey, On Devaney's Definition of Chaos, Amer. Math. Monthlly 99, (1992),332-334.

[BHM02] F. Blanchard, B. Host and A. Maass, Topological complexity, Ergod. Th. and Dynam. Sys. 20, (2000), 641-662.

[BHR] F. Blanchard, B. Host and S. Ruette, Asymptotic pairs in positive-entropy systems, Ergodic Theory Dynam. Systems, 22, (2002), 671-686.

[BGKM02] F. Blanchard, E. Glasner, S. Kolyada and A. Maass, On Li-Yorke pairs, J. Reine Angew. Math. 547, (2002), 51-68.

[Bro79] I. U. Bronstein, Extensions of minimal transformation groups, Sijthoff \& Noordhoff, 1979 .

[EGS75] R. Ellis, S. Glasner and L. Shapiro, Proximal-Isometric Flows, Advances in Math 17, (1975), 213-260.

[D89] R. Devaney, Chaotic dynamical systems, 2d edn. New York: Addison-Wesley (1989).

[F63] H. Furstenberg, The structure of distal flows, Amer. J. Math., 85, (1963), 477-515.

[F67] H. Furstenberg, Disjointness in ergodic theory, minimal sets, and a problem in Diophantine approximation, Math. Systems Theory, 1, (1967), 1-49.

[F81] H. Furstenberg, Recurrence in ergodic theory and combinatorial number theory, Princeton Univ. Press, 1981.

[E73] R. Ellis, The Veech structure theorem, Trans. Amer. Math. Soc. 186, (1973), 203-218.

[G76] E. Glasner, Proximal flows, Lecture Notes in Math. 517, Springer-Verlag, 1976.

[G03] E. Glasner, Ergodic Theory via Joinings, Mathematical Surveys and Monographs 101, AMS, Providence, RI, 2003.

[G05] E. Glasner, Topological weak mixing and Quasi-Bohr systems, Probability in mathematics, Israel J. Math., 148, (2005), 277-304.

[GM89] E. Glasner and D. Maon, Rigidity in topological dynamics, Ergod. Th. Dynam. Sys. 9, (1989), 309-320.

[GW79] E. Glasner and B. Weiss, On the construction of minimal skew-products, Israel J. of Math. 34, (1979), 321-336.

[GW93] E. Glasner and B. Weiss, Sensitive dependence on initial conditions, Nonlinearity, 6, (1993), 1067-1075.

[GY08] E. Glasner and X. Ye, Local entropy theory, to appear in Ergodic theory and Dynam. Sys.

[HY02] W. Huang and X.D. Ye, Devaney's chaos or 2-scattering implies Li-Yorke's chaos, Topology Appl., 117, (2002), 259-272.

[189] A. Iwanik, Independent sets of transitive points, Dynamical Systems and Ergodic Theory, Banach Center Publications, 23, (1989), 277-282.

[K73] K. Kuratowski, Applications of the Baire-category method to the problem of independent sets, Fund. Math., 81, (1973), 65-72.

[LY75] T. Y. Li and J. A. Yorke Period three implies chaos, Amer. Math. Monthly 82 (1975), 985-992.

[Mai04] J. H. Mai, Devaney's Chaos implies existence of s-scrambled sets, Proc. Amer. Math. Soc., 132, (2004), 2761-2767.

[Mar61] E. Marczewski, Independence and homomorphisms in abstract algebra, Fund. Math., 50, (1961), 45-61.

[MP80] N. Markley and G. M. E. Paul, Almost automorphic symbolic minimal sets without unique ergodicity, Israel J. Math. 34, (1979), (1980), 259-272.

[Mc76] D. C. McMahon, Weak mixing and a note on a structure theorem for minimal transformation groups, Illinois J. Math. 20, (1976), 186-197. 
[M64] J. Mycielski, Independent sets in topological algebras, Fund. Math., 55, (1964), 139147.

[V70] W. A. Veech, Point-distal flows, Amer. J. Math., 92, (1970), 205-242.

[V77] W. A. Veech, Topological dynamics, Bull. Amer. Math. Soc. 83, (1977), 775-830.

[W84] S. Williams, Toeplitz minimal flows which are not uniquely ergodic, Z. Wahrsch. Verw. Gebiete, 67, (1984), 95-107.

[Wo82] J. van der Woude, Topological dynamics, Dissertation, Vrije Universiteit, Amsterdam, 1982. CWI Tract, 22.

[Wo85] J. van der Woude, Characterizations of (H)PI extensions, Pacific J. of Math., 120, (1985), 453-467.

Mathematics Department, The City College, 137 Street and Convent Avenue, New York City, NY 10031, USA

E-mail address: ethanakin@earthlink.net

Department of Mathematics, Tel Aviv University, Tel Aviv, Israel

E-mail address: glasner@math.tau.ac.il

Department of Mathematics, University of Science and Technology of China, Hefei, Anhui, 230026, P.R. China

E-mail address: wenh@mail.ustc.edu.cn

E-mail address: songshao@ustc.edu.cn

E-mail address: yexd@ustc.edu.cn 